\title{
Basic Hypergeometric Functions as Limits of Elliptic Hypergeometric Functions ${ }^{\star}$
}

\author{
Fokko J. VAN DE BULT and Eric M. RAINS \\ MC 253-37, California Institute of Technology, 91125, Pasadena, CA, USA \\ E-mail:vdbult@caltech.edu,rains@caltech.edu
}

Received February 01, 2009; Published online June 10, 2009

doi:10.3842/SIGMA.2009.059

\begin{abstract}
We describe a uniform way of obtaining basic hypergeometric functions as limits of the elliptic beta integral. This description gives rise to the construction of a polytope with a different basic hypergeometric function attached to each face of this polytope. We can subsequently obtain various relations, such as transformations and three-term relations, of these functions by considering geometrical properties of this polytope. The most general functions we describe in this way are sums of two very-well-poised ${ }_{10} \phi_{9}$ 's and their Nassrallah-Rahman type integral representation.
\end{abstract}

Key words: elliptic hypergeometric functions, basic hypergeometric functions, transformation formulas

2000 Mathematics Subject Classification: 33D15

\section{Introduction}

Hypergeometric functions have played an important role in mathematics, and have been much studied since the time of Euler and Gauß. One of the goals of this research has been to obtain hypergeometric identities, such as evaluation and transformation formulas. Such formulas are of interest due to representation-theoretical interpretations, as well as their use in simplifying sums appearing in combinatorics.

In more recent times people have been trying to understand the structure behind these formulas. In particular people have studied the symmetry groups associated to certain hypergeometric functions, or the three terms relations satisfied by them (see [8] and [9]).

Another recent development is the advent of elliptic hypergeometric functions. This defines a whole new class of hypergeometric functions, in addition to the ordinary hypergeometric functions and the basic hypergeometric functions. A nice recent overview of this theory is given in [18]. For several of the most important kinds of formulas for classical hypergeometric functions there exist elliptic hypergeometric analogues. It is well known that one obtains basic hypergeometric functions upon taking a limit in these elliptic hypergeometric functions. However a systematic description of all possible limits had not yet been undertaken.

In this article we provide such a description of limits, extending work by Stokman and the authors [1]. This description provides some extra insight into elliptic hypergeometric functions, as it indicates what relations for elliptic hypergeometric functions correspond to what kinds of relations for basic hypergeometric functions. Conversely we can now more easily tell for what kind of relations there have not yet been found proper elliptic hypergeometric analogues.

More importantly though, this description provides more insight into the structure of basic hypergeometric functions and their relations, in the form of a geometrical description of a large

${ }^{\star}$ This paper is a contribution to the Proceedings of the Workshop "Elliptic Integrable Systems, Isomonodromy Problems, and Hypergeometric Functions" (July 21-25, 2008, MPIM, Bonn, Germany). The full collection is available at http://www.emis.de/journals/SIGMA/Elliptic-Integrable-Systems.html 
class of functions and relations. All the results for basic hypergeometric functions we obtain can be shown to be limits of previously known relations satisfied by sums of two very-well-poised ${ }_{10} \phi_{9}$ 's and their Nassrallah-Rahman like integral representation. However, we would have been unable to place them in a geometrical picture as we do in this article without considering these functions as limits of an elliptic hypergeometric function.

In this article we focus on the (higher-order) elliptic beta integral [14]. For any $m \in \mathbb{Z}_{\geq 0}$ the function $E^{m}(t)$ is defined for $t \in \mathbb{C}^{2 m+6}$ satisfying the balancing condition

$$
\prod_{r=0}^{2 m+5} t_{r}=(p q)^{m+1}
$$

by the formula

$$
E^{m}(t)=\left(\prod_{0 \leq r<s \leq 2 m+5}\left(t_{r} t_{s} ; p, q\right)\right) \frac{(p ; p)(q ; q)}{2} \int_{\mathcal{C}} \frac{\prod_{r=0}^{2 m+5} \Gamma\left(t_{r} z^{ \pm 1}\right)}{\Gamma\left(z^{ \pm 2}\right)} \frac{d z}{2 \pi i z} .
$$

Here $\Gamma$ denotes the elliptic gamma function and is defined in Section 2, as are the $(p, q)$-shifted factorials $(x ; p, q)$.

Two important results for the elliptic beta integral are the existence of an evaluation formula for $E^{0}$ and the fact that $E^{1}$ is invariant under an action of the Weyl group $W\left(E_{7}\right)$ of type $E_{7}$ [10]. A more thorough discussion of the elliptic beta integral is provided in Section 3.

The main result of this paper is the following (see Theorems 5.2-5.4), and its analogues for $m=0, m>1$.

Theorem 1.1. Let $P$ denote the convex polytope in $\mathbb{R}^{8}$ with vertices

$$
e_{i}+e_{j}, \quad 0 \leq i<j \leq 7, \quad \frac{1}{2}\left(\sum_{r=0}^{7} e_{r}\right)-e_{i}-e_{j}, \quad 0 \leq i<j \leq 7 .
$$

Then for each $\alpha \in P$ the limit

$$
B_{\alpha}^{1}(u)=\lim _{p \rightarrow 0} E^{1}\left(p^{\alpha_{0}} u_{0}, \ldots, p^{\alpha_{7}} u_{7}\right)
$$

exists as a function of $u \in \mathbb{C}^{8}$ satisfying the balancing condition $\prod u_{r}=q^{2}$. Moreover, $B_{\alpha}^{1}$ depends only on the face of the polytope which contains $\alpha$ and is a function of the projection of $\log (u)$ to the space orthogonal to that face.

Remark 1.2. The polytope $P$ was studied in an unrelated context in [3], where it was referred to as the "Hesse polytope", as antipodal pairs of vertices are in natural bijection with the bitangents of a plane quartic curve.

As stated the theorem is rather abstract, but for each point in this polytope we have an explicit expression of the limit as either a basic hypergeometric integral, or a basic hypergeometric series, or a product of $q$-shifted factorials (and sometimes several of these options). A graph containing all these functions is presented in Appendix A. We also obtain geometrical descriptions of various relations between these limits $B_{\alpha}^{1}$.

Note that the vertices of the polytope are given by the roots satisfying $\rho \cdot u=1$ of the root system $R\left(E_{8}\right)=\left\{u \in \mathbb{Z}^{8} \cup\left(\mathbb{Z}^{8}+\rho\right) \mid u \cdot u=2\right\}$, where $\rho=\{1 / 2\}^{8}$. In particular, the Weyl group $W\left(E_{7}\right)=\operatorname{Stab}_{W\left(E_{8}\right)}(\rho)$ acts on the polytope in a natural way, which is consistent with the $W\left(E_{7}\right)$-symmetry of $E^{1}$. As an immediate corollary of this $W\left(E_{7}\right)$ invariance we obtain both the symmetries of the limit $B_{\alpha}^{1}$ (determined by the stabilizer in $W\left(E_{7}\right)$ of the face containing $\alpha$ ) and 
transformations relating different limits (determined by the orbits of the face $\alpha$ ). Special cases of these include many formulas found in Appendix III of Gasper and Rahman [6]. For example, they include Bailey's four term transformation of very-well-poised ${ }_{10} \phi_{9}$ 's (as a symmetry of the sum of two ${ }_{10} \phi_{9}$ 's), the Nassrallah-Rahman integral representation of a very-well-poised ${ }_{8} \phi_{7}$ (as a transformation between two different limits) and the expression of a very-well-poised ${ }_{8} \phi_{7}$ in terms of the sum of two ${ }_{4} \phi_{3}$ 's.

Three term relations involving the different basic hypergeometric functions can be obtained as limits of $p$-contiguous relations satisfied by $E^{1}$ (and geometrically correspond to triples of points in $P$ differing by roots of $E_{7}$ ), while the $q$-contiguous relations satisfied by $E^{1}$ reduce to the $(q-)$ contiguous relations satisfied by its basic hypergeometric limits. In particular, we see that these two qualitatively different kinds of formulas for basic hypergeometric functions are closely related: indeed, they are different limits of essentially the same elliptic identity!

A similar statement can be made for $E^{0}$, which leads to evaluation formulas of its basic hypergeometric limits. Special cases of these include Bailey's sum for a very-well-poised ${ }_{8} \phi_{7}$ and the Askey-Wilson integral evaluation.

We would like to remark that a similar analysis can be performed for multivariate integrals. In particular the polytopes we obtain here are the same as the polytopes we get for the multivariate elliptic Selberg integrals (previously called type II integrals) of $[4,5,10,11]$. In a future article the authors will also consider the limits of the (bi-)orthogonal functions of [10], generalizing and systematizing the $q$-Askey scheme.

The article is organized as follows. We begin with a small section on notations, followed by a review of some of the properties of the elliptic beta integrals. In Section 4 we will describe the explicit limits we consider. In Section 5 we define convex polytopes, each point of which corresponds to a direction in which we can take a limit. Moreover in this section we prove the main theorems of this article, describing some basic properties of these basic hypergeometric limits in terms of geometrical properties of the polytope. In Section 6 we harvest by considering the consequences in the case we know non-trivial transformations of the elliptic beta integral. Section 7 is then devoted to explicitly giving some of these consequences in an example, on the level of ${ }_{2} \phi_{1}$. Section 8 describes some peculiarities specific to the evaluation $\left(E^{0}\right)$ case. Finally in Section 9 we consider some remaining questions, in particular focusing on what happens for limits outside our polytope. The appendices give a graphical representation of the different limits we obtain and a quick way of determining what kinds of relations these functions satisfy.

\section{Notation}

Throughout the article $p$ and $q$ will be complex numbers satisfying $|p|,|q|<1$, in order to ensure convergence of relevant series and products. Note that $q$ is generally assumed to be fixed, while $p$ may vary.

We use the following notations for $q$-shifted factorials and theta functions:

$$
(x ; q)=(x ; q)_{\infty}=\prod_{j=0}^{\infty}\left(1-x q^{j}\right), \quad(x ; q)_{k}=\frac{(x ; q)_{\infty}}{\left(x q^{k} ; q\right)_{\infty}}, \quad \theta(x ; q)=(x, q / x ; q),
$$

where in the last equation we used the convention that $\left(a_{1}, \ldots, a_{n} ; q\right)=\prod_{i=1}^{n}\left(a_{i} ; q\right)$, which we will also apply to gamma functions. Moreover we will use the shorthand $\left(x z^{ \pm 1} ; q\right)=\left(x z, x z^{-1} ; q\right)$.

Many of the series we obtain as limits are confluent, and in some cases, highly confluent. To simplify the description of such limits, we will use a slightly modified version of the notation for 
basic hypergeometric series in [6]. In particular we set

$$
{ }_{r} \phi_{s}^{(n)}\left(\begin{array}{c}
a_{1}, a_{2}, \ldots, a_{r} \\
b_{1}, b_{2}, \ldots, b_{s}
\end{array} ; q, z\right)=\sum_{k=0}^{\infty} \frac{\left(a_{1}, a_{2}, \ldots, a_{r} ; q\right)_{k}}{\left(q, b_{1}, b_{2}, \ldots, b_{s} ; q\right)_{k}} z^{k}\left((-1)^{k} q^{\left(\begin{array}{c}
k \\
2
\end{array}\right)}\right)^{n+s+1-r} .
$$

In terms of the original ${ }_{r} \phi_{s}$ from [6] this is

$$
{ }_{r} \phi_{s}^{(n)}\left(\begin{array}{c}
a_{1}, a_{2}, \ldots, a_{r} \\
b_{1}, b_{2}, \ldots, b_{s}
\end{array} ; q, z\right)= \begin{cases}{ }_{r} \phi_{s+n}\left(\begin{array}{c}
a_{1}, a_{2}, \ldots, a_{r} \\
b_{1}, b_{2}, \ldots, b_{s}, \underbrace{0, \ldots, 0} ; q, z)
\end{array}\right. & \text { if } n>0, \\
r \phi_{s}\left(\begin{array}{c}
\left.a_{1}, a_{2}, \ldots, a_{r} ; q, z\right) \\
b_{1}, b_{2}, \ldots, b_{s}
\end{array}\right)^{n} n=0, \\
{ }_{r-n} \phi_{s}\left(\begin{array}{c}
a_{1}, a_{2}, \ldots, a_{r}, \overbrace{0, \ldots, 0}^{-n} \\
b_{1}, b_{2}, \ldots, b_{s}
\end{array} q, z\right) & \text { if } n<0 .\end{cases}
$$

In the case $n=0$ we will of course in general omit the (0), as we then re-obtain the usual definition of ${ }_{r} \phi_{s}$. Moreover, when considering specific series, we will often omit the $r$ and $s$ from the notation as they can now be derived by counting the number of parameters. We also extend the definition of very-well-poised series in this way:

$$
{ }_{r} W_{r-1}^{(n)}\left(a ; b_{1}, \ldots, b_{r-3} ; q, z\right)={ }_{r} \phi_{r-1}^{(n)}\left(\begin{array}{c}
a, \pm q \sqrt{a}, b_{1}, \ldots, b_{r-3} \\
\pm \sqrt{a}, a q / b_{1}, \ldots, a q / b_{r-3}
\end{array} ; q, z\right) .
$$

Note, however, that this function cannot be obtained simply by setting some parameters to 0 in the usual very-well-poised series. Indeed, setting the parameter $b$ to zero in a very-well-poised series causes the corresponding parameter $a q / b$ to become infinite, making the limit fail. For the basic hypergeometric bilateral series we use the usual notation

$$
{ }_{r} \psi_{r}\left(\begin{array}{c}
a_{1}, \ldots, a_{r} \\
b_{1}, \ldots b_{r}
\end{array} ; q, z\right)=\sum_{k \in \mathbb{Z}} \frac{\left(a_{1}, \ldots, a_{r} ; q\right)_{k}}{\left(b_{1}, \ldots, b_{r} ; q\right)_{k}} z^{k}
$$

We define $p, q$-shifted factorials by setting

$$
(z ; p, q)=\prod_{j, k \geq 0}\left(1-p^{j} q^{k} z\right)
$$

The elliptic gamma function [13] is defined by

$$
\Gamma(z)=\Gamma(z ; p, q)=\frac{(p q / z ; p, q)}{(z ; p, q)}=\prod_{j, k=0}^{\infty} \frac{1-p^{j+1} q^{k+1} / z}{1-p^{j} q^{k} z} .
$$

We omit the $p$ and $q$ dependence whenever this does not cause confusion. Note that the elliptic gamma function satisfies the difference equations

$$
\Gamma(q z)=\theta(z ; p) \Gamma(z), \quad \Gamma(p z)=\theta(z ; q) \Gamma(z)
$$

and the reflection equation

$$
\Gamma(z) \Gamma(p q / z)=1
$$




\section{$3 \quad$ Elliptic beta integrals}

In this section we introduce the elliptic beta integrals and we recall their relevant properties. As a generalization of Euler's beta integral evaluation, the elliptic beta integral was introduced by Spiridonov in [14]. An extension by two more parameters was shown to satisfy a transformation formula $[15,10]$, corresponding to a symmetry with respect to the Weyl group of $E_{7}$. We can generalize the beta integral by adding even more parameters, but unfortunately not much is known about these integrals, beyond some quadratic transformation formulas for $m=2$ [12] and a transformation to a multivariate integral [10].

Definition 3.1. Let $m \in \mathbb{Z}_{\geq 0}$. Define the set $\mathcal{H}_{m}=\left\{z \in \mathbb{C}^{2 m+6} \mid \prod_{i} z_{i}=(p q)^{m+1}\right\} / \sim$, where $\sim$ is the equivalence relation induced by $z \sim-z$. For parameters $t \in \mathcal{H}_{m}$ we define the renormalized elliptic beta integral by

$$
E^{m}(t)=\left(\prod_{0 \leq r<s \leq 2 m+5}\left(t_{r} t_{s} ; p, q\right)\right) \frac{(p ; p)(q ; q)}{2} \int_{\mathcal{C}} \frac{\prod_{r=0}^{2 m+5} \Gamma\left(t_{r} z^{ \pm 1}\right)}{\Gamma\left(z^{ \pm 2}\right)} \frac{d z}{2 \pi i z},
$$

where the integration contour $\mathcal{C}$ circles once around the origin in the positive direction and separates the poles at $z=t_{r} p^{j} q^{k}\left(0 \leq r \leq 2 m+5\right.$ and $\left.j, k \in \mathbb{Z}_{\geq 0}\right)$ from the poles at $z=t_{r}^{-1} p^{-j} q^{-k}$ $\left(0 \leq r \leq 2 m+5\right.$ and $\left.j, k \in \mathbb{Z}_{\geq 0}\right)$. For parameters $t$ for which such a contour does not exist (i.e. if $t_{r} t_{s} \in p^{\mathbb{Z}_{\leq 0}} q^{\mathbb{Z} \leq 0}$ ) we define $E^{m}$ to be the analytic continuation of the function to these parameters.

Observe that this function is well-defined, in the sense that $E^{m}(t)=E^{m}(-t)$ by a change of integration variable $z \rightarrow-z$. We can choose the contour in (3.1) to be the unit circle itself whenever $\left|t_{r}\right|<1$ for all $r$. If $t_{r} t_{s}=p^{-n_{1}} q^{-n_{2}}$ for some $n_{1}, n_{2} \geq 0, r \neq s$, then the desired contour fails to exist, but we can obtain the analytic continuation by picking up residues of offending poles before specializing the parameter $t$. In particular the prefactor $\prod_{0 \leq r<s \leq 2 m+5}\left(t_{r} t_{s} ; p, q\right)$ cancels all the poles of these residues and thus ensures $E^{m}$ is analytic at those points. In this case the integral reduces to a finite sum. Indeed for $t_{0} t_{1}=p^{-n_{1}} q^{-n_{2}}$, we have

$$
\begin{aligned}
E^{m}(t)= & \left(p q / t_{0} t_{1} ; p, q\right)\left(\prod_{\substack{0 \leq r<s \leq 2 m+5 \\
(r, s) \neq(0,1)}}\left(t_{r} t_{s} ; p, q\right)\right) \Gamma\left(p q t_{0}^{2}, t_{1} / t_{0}\right) \prod_{r=2}^{2 m+5} \Gamma\left(t_{r} t_{0}^{ \pm 1}\right) \\
& \times \sum_{k=0}^{n_{1}} \prod_{r=0}^{2 m+5} \frac{\theta\left(t_{r} t_{0} ; q, p\right)_{k}}{\theta\left(p q t_{0} / t_{r} ; q, p\right)_{k}} \frac{\theta\left(p q t_{0}^{2} ; q, p\right)_{2 k}}{\theta\left(t_{0}^{2} ; q, p\right)_{2 k}} \sum_{l=0}^{n_{2}} \prod_{r=0}^{2 m+5} \frac{\theta\left(t_{r} t_{0} ; p, q\right)_{l}}{\theta\left(p q t_{0} / t_{r} ; p, q\right)_{l}} \frac{\theta\left(p q t_{0}^{2} ; p, q\right)_{2 l}}{\theta\left(t_{0}^{2} ; p, q\right)_{2 l}},
\end{aligned}
$$

where we use the notation $\theta(x ; q, p)_{k}=\prod_{r=0}^{k-1} \theta\left(x p^{r} ; q\right)$. There are other singular cases, more difficult to evaluate, but in general $E^{m}(t)$ is analytic on all of $\mathcal{H}_{m}$, as follows from [10, Lemma 10.4].

The elliptic beta integral evaluation of [14] is now given by

Theorem 3.2. For $t \in \mathcal{H}_{0}$ we have

$$
E^{0}(t)=\prod_{0 \leq r<s \leq 5}\left(p q / t_{r} t_{s} ; p, q\right)
$$

Apart from in [14], elementary proofs of this theorem are given in [17] and [10]. Moreover in [10] several multivariate extensions of this result are presented.

A second important result is the $E_{7}$ symmetry satisfied by $E^{1}$. Before we can state this in a theorem we first have to introduce the Weyl groups and their actions. 
Definition 3.3. Let $\rho \in \mathbb{R}^{8}$ be the vector $\rho=(1 / 2, \ldots, 1 / 2)$. Define the root system $R\left(E_{8}\right)$ of $E_{8}$ by $R\left(E_{8}\right)=\left\{v \in \mathbb{Z}^{8} \cup\left(\mathbb{Z}^{8}+\rho\right) \mid v \cdot v=2\right\}$. Moreover the root system $R\left(E_{7}\right)$ of $E_{7}$ is given by $R\left(E_{7}\right)=\left\{v \in R\left(E_{8}\right) \mid v \cdot \rho=0\right\}$. Denote by $s_{\alpha}$ the reflection in the hyperplane orthogonal to $\alpha$ (i.e. $s_{\alpha}(\beta)=\beta-(\alpha \cdot \beta) \alpha$ for $\alpha \in R\left(E_{8}\right)$ ). The corresponding Weyl group $W\left(E_{7}\right)$ is the reflection group generated by $\left\{s_{\alpha} \mid \alpha \in R\left(E_{7}\right)\right\}$. Apart from the natural action of $E_{7}$ on $\mathbb{R}^{8}$, we need the action on $\mathcal{H}_{1}$ given by $w t=\exp (w(\log (t)))$ for $t \in \mathcal{H}_{1}$ (where $\log \left(\left(t_{0}, \ldots, t_{7}\right)\right)=\left(\log \left(t_{0}\right), \ldots, \log \left(t_{7}\right)\right)$ and similarly for exp). Finally we will often meet the $W\left(E_{7}\right)$ orbit $S$ in $R\left(E_{8}\right)$ given by $S=\left\{v \in R\left(E_{8}\right) \mid s \cdot \rho=1\right\}$.

Note that the action of $W\left(E_{7}\right)$ on $\mathcal{H}_{1}$ is well-defined due to the equivalence of $t \sim-t$. Indeed, if we reflect in a root of the form $\rho-e_{i}-e_{j}-e_{k}-e_{l}$ then we have to take square roots of the $t_{j}$, but if we do this consistently ( $\operatorname{such}$ that $\prod_{j} \sqrt{t_{j}}=p q$ ), the final result will differ at most by a factor -1 . A more thorough analysis of this action is given in [1].

Now we can formulate the following theorem describing the transformations satisfied by $E^{1}$ (see [15] and [10], the latter containing also a multivariate extension).

Theorem 3.4. The integral $E^{1}$ is invariant under the action of $W\left(E_{7}\right)$, i.e. for all $w \in W\left(E_{7}\right)$ and $t \in \mathcal{H}_{1}$ we have $E^{1}(t)=E^{1}(w t)$.

In the cited references the transformation has certain products of elliptic gamma functions on one or both sides of the equation, but these factors are precisely canceled by our choice of prefactor.

Let us recall the following contiguous relations satisfied by $E^{1}$ [14] (it is shown there for $m=0$, but the proof is identical to that of the $m=1$ case, apart from the use of the Weyl group action). We have rewritten it in a clearly $W\left(E_{7}\right)$ invariant form.

Theorem 3.5. Let us denote $t^{\rho}=\prod_{j} t_{j}^{\rho_{j}}$, and $t \cdot p^{\rho}=\left(t_{0} p^{\rho_{0}}, \ldots, t_{7} p^{\rho_{7}}\right)$. Then if $\alpha, \beta, \gamma \in R\left(E_{7}\right)$ form an equilateral triangle (i.e. $\alpha \cdot \beta=\alpha \cdot \gamma=\beta \cdot \gamma=1$ ) we have

$$
\begin{aligned}
& \prod_{\substack{\delta \in S \\
\delta \cdot(\alpha-\beta)=\delta \cdot(\alpha-\gamma)=1}}\left(t^{\delta} p^{\delta \cdot \beta} ; q\right) t^{\gamma} \theta\left(t^{\beta-\gamma} ; q\right) E^{1}\left(t \cdot p^{\alpha}\right) \\
+ & \prod_{\substack{\delta \in S \\
\delta \cdot(\beta-\gamma)=\delta \cdot(\beta-\alpha)=1}}\left(t^{\delta} p^{\delta \cdot \gamma} ; q\right) t^{\alpha} \theta\left(t^{\gamma-\alpha} ; q\right) E^{1}\left(t \cdot p^{\beta}\right) \\
+ & \prod_{\substack{\delta \in S \\
\delta \cdot(\gamma-\alpha)=\delta \cdot(\gamma-\beta)=1}}\left(t^{\delta} p^{\delta \cdot \alpha} ; q\right) t^{\beta} \theta\left(t^{\alpha-\beta} ; q\right) E^{1}\left(t \cdot p^{\gamma}\right)=0 .
\end{aligned}
$$

Proof. Observe that the relation is satisfied by the integrands when $\alpha=e_{1}-e_{0}, \beta=e_{2}-e_{0}$ and $\gamma=e_{3}-e_{0}$, where $\left\{e_{i}\right\}$ form the standard orthonormal basis of $\mathbb{R}^{8}$, due to the fundamental relation

$$
\frac{1}{y} \theta\left(w x^{ \pm 1}, y z^{ \pm 1} ; q\right)+\frac{1}{z} \theta\left(w y^{ \pm 1}, z x^{ \pm 1} ; q\right)+\frac{1}{x} \theta\left(w z^{ \pm 1}, x y^{ \pm 1} ; q\right)=0 .
$$

Integrating the identity now proves the contiguous relations for these special $\alpha, \beta$ and $\gamma$. As the equation is invariant under the action of $W\left(E_{7}\right)$, which acts transitively on the set of all equilateral triangles of roots, the result holds for all such triangles.

These contiguous relations can be combined to obtain relations of three $E^{1}$ 's which differ by shifts along any vector in the root lattice of $E_{7}$ (i.e., the smallest 7-dimensional lattice in $\mathbb{R}^{8}$ containing $R\left(E_{7}\right)$ ). In particular the equation relating $E^{1}\left(t \cdot p^{\alpha}\right), E^{1}(t)$ and $E^{1}\left(t \cdot p^{-\alpha}\right)$ for $\alpha=e_{1}-e_{0}$ is the elliptic hypergeometric equation studied by Spiridonov in, amongst others, [16]. 


\section{Limits to basic hypergeometric functions}

In order to obtain basic hypergeometric limits from these integrals we let $p \rightarrow 0$. As our parameters can not be chosen independently of $p$ (due to the balancing condition), we have to explicitly describe how they behave as $p \rightarrow 0$. Different ways the parameters depend on $p$ require different ways of obtaining the limit. In this section we describe the different limits of interest to us.

Using the notation of Theorem 3.5 we see that $u \cdot p^{\alpha}$, for $u$ independent of $p$, is an element of $\mathcal{H}_{m}$ if $\alpha \in \mathbb{R}^{2 m+6}$ with $\sum_{r} \alpha_{r}=m+1$, and $u \in \tilde{\mathcal{H}}_{m}=\left\{z \in \mathbb{C}^{2 m+6} \mid \prod_{i} z_{i}=q^{m+1}\right\} / \sim$ (where we again have $z \sim-z$ ). In particular in this section we will describe various conditions on $\alpha$ which ensure that the limit

$$
B_{\alpha}^{m}(u)=\lim _{p \rightarrow 0} E^{m}\left(u \cdot p^{\alpha}\right)
$$

is well-defined, and give explicit expressions for this limit. In particular, for $m=1$ we would like such expressions for $\alpha$ in the entire Hesse polytope as defined in Theorem 1.1.

The simplest way to obtain a limit is given by the following proposition.

Proposition 4.1. For $\alpha \in \mathbb{R}^{2 m+6}$ satisfying $\sum_{r} \alpha_{r}=m+1$ and such that $0 \leq \alpha_{r} \leq 1$ for all $r$, the limit in (4.1) exists and we have

$$
B_{\alpha}^{m}(u)=\prod_{\substack{0 \leq r<s \leq 2 m+5 \\ \alpha_{r}=\alpha_{s}=0}}\left(u_{r} u_{s} ; q\right) \frac{(q ; q)}{2} \int_{\mathcal{C}}\left(z^{ \pm 2} ; q\right) \frac{\prod_{r: \alpha_{r}=1}\left(q / u_{r} z^{ \pm 1} ; q\right)}{\prod_{r: \alpha_{r}=0}\left(u_{r} z^{ \pm 1} ; q\right)} \frac{d z}{2 \pi i z}
$$

where the contour is a deformation of the unit circle which separates the poles at $z=u_{r} q^{n}$ $\left(\alpha_{r}=0, n \geq 0\right)$ from those at $u_{r}^{-1} q^{-n}\left(\alpha_{r}=0, n \geq 0\right)$.

We want to stress that the limit also exists if the integral above is not well-defined (i.e. when there exists no proper contour, when $u_{r} u_{s}=q^{-n}$ for some $\alpha_{r}=\alpha_{s}=0$ ). In that case the limit $B_{\alpha}^{m}$ is equal to the analytic continuation of the integral representation to these values of the parameters.

Proof. Observe that we can determine limits of the elliptic gamma function by

$$
\lim _{p \rightarrow 0} \Gamma\left(p^{\gamma} z\right)= \begin{cases}\frac{1}{(z ; q)} & \text { if } \gamma=0 \\ 1 & \text { if } 0<\gamma<1 \\ (q / z ; q) & \text { if } \gamma=1 .\end{cases}
$$

In fact $\Gamma\left(p^{\gamma} z\right)$ is well-defined and continuous in $p$ at $p=0$ for $0 \leq \gamma \leq 1$. These limits can thus be obtained by just plugging in $p=0$. Similarly observe that

$$
\lim _{p \rightarrow 0}\left(p^{\gamma} z ; p, q\right)= \begin{cases}(z ; p, q) & \text { if } \gamma=0 \\ 1 & \text { if } \gamma>0\end{cases}
$$

The result now follows from noting that an integration contour which separates the poles at $z=u_{r} q^{n}\left(\alpha_{r}=0, n \geq 0\right)$ from those at $u_{r}^{-1} q^{-n}\left(\alpha_{r}=0, n \geq 0\right)$ will also work in the definition of $E_{m}\left(u \cdot p^{\alpha}\right)$ if $p$ is small enough (as the poles of the integrand created by $u_{r}$ 's with $\alpha_{r}>0$ will all converge either to 0 or to infinity; in particular they will remain on the correct side of the contour for small enough $p$ ). Thus we can just plug in $p=0$ in the integral to obtain the limit.

This proof only works when the parameters $u$ are such that there exists a contour for the limiting integral. However, this implies these limits work outside a finite set of co-dimension 
one divisors. Indeed, on compacta outside these divisors the convergence is uniform. Using the Stieltjes-Vitali theorem we can conclude that the limit also holds on these divisors, and is in fact uniform on compacta of the entire parameter space. Moreover Stieltjes-Vitali tells us that the limit function is analytic in these points as well.

A second kind of limit, following $[11, \S 5]$, can be obtained by first breaking the symmetry of the integrand. This leads to the following proposition.

Proposition 4.2. Let $\alpha \in \mathbb{R}^{2 m+6}$ satisfy $\sum_{r} \alpha_{r}=m+1$ and $\alpha_{0} \leq \alpha_{1} \leq \alpha_{2}$. Define $\beta=$ $\alpha_{0}+\alpha_{1}+\alpha_{2}$ and impose the extra conditions $\beta \leq \alpha_{r} \leq-\beta$ for $r=0,1,2$ and $-\beta \leq \alpha_{r} \leq 1+\beta$ for $r \geq 3$. Then the limit in (4.1) exists, and takes one of the following forms:

- If $\alpha_{0}=\alpha_{1}=-\alpha_{2}$ (thus $\left.\beta=\alpha_{0}\right)$, then

$$
\begin{aligned}
B_{\alpha}^{m}(t)= & \frac{\prod_{r \geq 3: \alpha_{r}=-\alpha_{0}}\left(u_{r} u_{0}, u_{r} u_{1} ; q\right)}{\left(q / u_{0} u_{2}, q / u_{1} u_{2} ; q\right)}\left(u_{0} u_{1} ; q\right)^{1_{\left\{\alpha_{0}=-1 / 2\right\}}} \\
& \times(q ; q) \int_{\mathcal{C}} \theta\left(u_{0} u_{1} u_{2} / z ; q\right) \frac{\left(q / u_{2} z ; q\right)}{\left(u_{0} / z, u_{1} / z ; q\right)} \\
& \times \frac{\prod_{r \geq 3: \alpha_{r}=1+\alpha_{0}}\left(q z / u_{r} ; q\right)}{\prod_{r \geq 3: \alpha_{r}=-\alpha_{0}}\left(u_{r} z ; q\right)}\left(\frac{\left(1-z^{2}\right)\left(q z / u_{2} ; q\right)}{\left(u_{0} z, u_{1} z ; q\right)}\right)^{1_{\left\{\alpha_{0}=-1 / 2\right\}}} \frac{d z}{2 \pi i z}
\end{aligned}
$$

where the contour separates the downward from the upward pole sequences. Here $1_{\left\{\alpha_{0}=-1 / 2\right\}}$ equals 1 if $\alpha_{0}=-1 / 2$ and 0 otherwise.

- If $\alpha_{0}<\alpha_{1}=-\alpha_{2}\left(\right.$ again $\left.\beta=\alpha_{0}\right)$, then

$$
\begin{aligned}
B_{\alpha}^{m}(u)= & \frac{(q ; q)}{\left(q / u_{1} u_{2} ; q\right)} \prod_{\substack{3 \leq r \leq 2 m+5 \\
\alpha_{r}=-\alpha_{0}}}\left(u_{r} u_{0} ; q\right) \int_{\mathcal{C}} \theta\left(u_{0} u_{1} u_{2} / z ; q\right) \\
& \times \frac{1}{\left(u_{0} / z ; q\right)} \frac{\prod_{r \geq 3: \alpha_{r}=1+\alpha_{0}}\left(q z / u_{r} ; q\right)}{\prod_{r \geq 3: \alpha_{r}=-\alpha_{0}}\left(u_{r} z ; q\right)}\left(\frac{\left(1-z^{2}\right)}{\left(u_{0} z ; q\right)}\right)^{\left.1_{\left\{\alpha_{0}=-1 / 2\right.}\right\}} \frac{d z}{2 \pi i z},
\end{aligned}
$$

where the contour separates the downward poles from the upward ones.

- Finally, if $\alpha_{1}<-\alpha_{2}$ (thus $\beta<\alpha_{0}$ ), then

$$
B^{m}(t)=(q ; q) \int_{\mathcal{C}} \theta\left(u_{0} u_{1} u_{2} / z ; q\right) \frac{\prod_{r: \alpha_{r}=1+\beta}\left(q z / u_{r} ; q\right)}{\prod_{r: \alpha_{r}=-\beta}\left(u_{r} z ; q\right)}\left(1-z^{2}\right)^{1_{\{\beta=-1 / 2\}}} \frac{d z}{2 \pi i z},
$$

where the contour excludes the poles but circles the essential singularity at zero.

Proof. In order to obtain these limits we will break the symmetry of the integral. We first rewrite (3.4) in the form

$$
\frac{\theta\left(s_{0} s_{1} s_{2} / z, s_{0} z, s_{1} z, s_{2} z ; q\right)}{\theta\left(z^{2}, s_{0} s_{1}, s_{0} s_{2}, s_{1} s_{2} ; q\right)}+\left(z \leftrightarrow z^{-1}\right)=1
$$

Since the integrand of $E^{m}$ is invariant under the interchange of $z \rightarrow z^{-1}$, we can multiply by the left hand side of the above equation and observe that the integrand splits in two parts, each 
integrating to the same value. Therefore, the integral itself is equal to twice the integral of either part, and we thus obtain

$$
\begin{aligned}
E^{m}(t)= & \prod_{0 \leq r<s \leq 2 m+5}\left(t_{r} t_{s} ; p, q\right)(p ; p)(q ; q) \\
& \times \int_{\mathcal{C}} \frac{\prod_{r=0}^{2 m+5} \Gamma\left(t_{r} z^{ \pm 1}\right)}{\Gamma\left(z^{ \pm 2}\right)} \frac{\theta\left(s_{0} s_{1} s_{2} / z, s_{0} z, s_{1} z, s_{2} z ; q\right)}{\theta\left(z^{2}, s_{0} s_{1}, s_{0} s_{2}, s_{1} s_{2} ; q\right)} \frac{d z}{2 \pi i z} .
\end{aligned}
$$

The poles introduced by the factor $1 / \theta\left(z^{2} ; q\right)$ are canceled by zeros of the factor $1 / \Gamma\left(z^{ \pm 2}\right)$, as we have

$$
\frac{1}{\Gamma\left(z^{ \pm 2}\right) \theta\left(z^{2} ; q\right)}=\frac{\Gamma\left(p q z^{2}\right)}{\Gamma\left(p z^{2}\right)}=\theta\left(p z^{2} ; p\right)=\theta\left(z^{-2} ; p\right)
$$

using the difference and reflection equations satisfied by the elliptic gamma functions. This process therefore does not introduce any extra poles to the integrand; we may therefore use the same contour as before. In fact, since some of the original poles might have been cancelled, the constraints on the contour can be correspondingly weakened.

Now, specialize $s_{r}=t_{r}(r=0,1,2)$ in $(4.2)$ and simplify to obtain

$$
\begin{aligned}
E^{m}(t)= & \frac{\prod_{0 \leq r<s \leq 2}\left(p t_{r} t_{s} ; p, q\right) \prod_{r=0}^{2} \prod_{s=3}^{2 m+5}\left(t_{r} t_{s} ; p, q\right) \prod_{3 \leq r<s \leq 2 m+5}\left(t_{r} t_{s} ; p, q\right)}{\left(q / t_{0} t_{1}, q / t_{0} t_{2}, q / t_{1} t_{2} ; q\right)} \\
& \times(p ; p)(q ; q) \int_{\mathcal{C}} \theta\left(z^{-2} ; p\right) \theta\left(t_{0} t_{1} t_{2} / z ; q\right) \prod_{r=0}^{2} \Gamma\left(p t_{r} z, t_{r} / z\right) \prod_{r=3}^{2 m+5} \Gamma\left(t_{r} z^{ \pm 1}\right) \frac{d z}{2 \pi i z} .
\end{aligned}
$$

Now change the integration variable $z \rightarrow z p^{\beta}$. The inequalities $\alpha_{0}, \alpha_{1}, \alpha_{2} \geq \beta$ and $-\beta \leq \alpha_{r}$, $3 \leq r$ ensure that the downward poles remain bounded and the upward poles remain bounded away from 0 as $p \rightarrow 0$. There thus (for generic $u_{r}$ ) exists a contour valid for all sufficiently small $p$. After fixing such a contour, the limit again follows by simply plugging in $p=0$; the constraints on $\alpha$ are necessary and sufficient to ensure that all gamma functions in the integrand have well-defined limits.

The two previous limits still do not allow us to take limits for each possible vector in the Hesse polytope (in the $m=1$ case). Indeed (as we will show below) we have covered the polytope, modulo the action of $S_{8}$ to sort the entries $\alpha_{0} \leq \cdots \leq \alpha_{7}$, as long as either $\alpha_{0} \geq 0$ (Proposition 4.1) or $\alpha_{1}+\alpha_{2} \leq 0$ (Proposition 4.2). The remaining limits require a more careful look and are given by the following proposition.

Proposition 4.3. Let $\alpha \in \mathbb{R}^{2 m+6}$ satisfy $\sum_{r} \alpha_{r}=m+1$ and assume $-1 / 2 \leq \alpha_{0}<0$, $\alpha_{0} \leq$ $\alpha_{1} \leq \alpha_{2} \leq \cdots \leq \alpha_{m+3} \leq 1+\alpha_{0}$ and for $2 \leq k \leq m+3$,

$$
\sum_{r \in I}\left(\alpha_{r}+\alpha_{0}\right) \geq 2 \alpha_{0}, \quad I \subset\{1,2, \ldots, 2 m+5\}, \quad|I|=k
$$

hold. Then the limit in (4.1) exists.

- If $\alpha_{0}=\alpha_{1}=-1 / 2\left(\right.$ thus $\left.\alpha_{2}=\cdots=\alpha_{2 m+5}=1 / 2\right)$ we have

$$
\begin{aligned}
B_{\alpha}^{m}(u)= & \frac{\prod_{r=2}^{2 m+5}\left(u_{r} u_{1}, q u_{0} / u_{r} ; q\right)}{\left(q u_{0}^{2}, u_{0} u_{1}, u_{1} / u_{0} ; q\right)} \\
& \times{ }_{2 m+8} W_{2 m+7}\left(u_{0}^{2} ; u_{0} u_{1}, u_{0} u_{2}, \ldots, u_{0} u_{2 m+5} ; q, q\right)+\left(u_{0} \leftrightarrow u_{1}\right) .
\end{aligned}
$$


- If $\alpha_{0}=-1 / 2<\alpha_{1}$, and if $\alpha_{1}+\alpha_{2}=0$ the extra condition $\left|u_{1} u_{2}\right|<1$, we have with $n=\#\left\{r: \alpha_{r}<1 / 2\right\}-3$

$$
B_{\alpha}^{m}(u)=\frac{\left\{\left(u_{1} u_{2} ; q\right)\right\} \prod_{r: \alpha_{r}=1 / 2}\left(q u_{0} / u_{r} ; q\right)}{\left(q u_{0}^{2} ; q\right)} W^{(n)}\left(u_{0}^{2} ; u_{0} u_{r}: \alpha_{r}=1 / 2 ; q, u_{0}^{n} \prod_{r>0: \alpha_{r}<1 / 2} u_{r}\right),
$$

where the notation implies we take as parameters $u_{0} u_{r}$ for those $r$ which satisfy $\alpha_{r}=1 / 2$ and the factor $\left(u_{1} u_{2} ; q\right)$ appears only if $\alpha_{1}+\alpha_{2}=0$.

- If $-1 / 2<\alpha_{0}=\alpha_{1}<0$ then

$$
\begin{aligned}
B_{\alpha}^{m}(u)= & \frac{\prod_{\alpha_{r}=-\alpha_{0}}\left(u_{1} u_{r} ; q\right) \prod_{\alpha_{r}=1+\alpha_{0}}\left(q u_{0} / u_{r} ; q\right)}{\left(u_{1} / u_{0} ; q\right)} \\
& \times \phi^{(n)}\left(\begin{array}{c}
u_{0} u_{r}: \alpha_{r}=-\alpha_{0} \\
q u_{0} / u_{1}, q u_{0} / u_{r}: \alpha_{r}=1+\alpha_{0}
\end{array} ; q, q\right)+\left(u_{0} \leftrightarrow u_{1}\right),
\end{aligned}
$$

where $n=\#\left\{r: \alpha_{r}=-\alpha_{0}\right\}-\#\left\{r: \alpha_{r}=1+\alpha_{0}\right\}-2$.

- If $-1<2 \alpha_{0}=\sum_{r \geq 1: \alpha_{r}+\alpha_{0}<0}\left(\alpha_{r}+\alpha_{0}\right)$ and $\alpha_{1}>\alpha_{0}$, and if $\alpha_{1}+\alpha_{2}=0$ the extra condition $\left|u_{1} u_{2}\right|<1$, we get

$$
\begin{aligned}
B_{\alpha}^{m}(u)= & \left\{\left(u_{1} u_{2} ; q\right)\right\} \prod_{r: \alpha_{r}=1+\alpha_{0}}\left(q u_{0} / u_{r} ; q\right) \\
& \times \phi^{(n)}\left(\begin{array}{c}
u_{0} u_{r}: \alpha_{r}=-\alpha_{0} \\
q u_{0} / u_{r}: \alpha_{r}=1+\alpha_{0}
\end{array} ; q, u_{0}^{-2} \prod_{r>0: \alpha_{r}<-\alpha_{0}}\left(u_{r} u_{0}\right)\right),
\end{aligned}
$$

where $n=\#\left\{r: \alpha_{r}<-\alpha_{0}\right\}-4-\#\left\{r: \alpha_{r}=1+\alpha_{0}\right\}+\#\left\{r: \alpha_{r}=-\alpha_{0}\right\}$, and the factor $\left(u_{1} u_{2} ; q\right)$ appears only if $\alpha_{1}+\alpha_{2}=0$.

- Finally if $2 \alpha_{0}<\sum_{r \geq 1: \alpha_{r}+\alpha_{0}<0}\left(\alpha_{r}+\alpha_{0}\right)$ we get

$$
B_{\alpha}^{m}(u)=\prod_{r: \alpha_{r}=1+\alpha_{0}}\left(q u_{0} / u_{r} ; q\right)
$$

Proof. Note that limits in the cases $\alpha_{0}=\alpha_{1}=-1 / 2$ and $-1 / 2<\alpha_{0}=\alpha_{1} \geq-\alpha_{r}(r \geq 2)$ are given in Proposition 4.2. Together with the limits in this proposition we have thus covered all of the possible values for $\alpha$ at least once.

Due to the condition $\alpha_{0}<0$, in the integral definition of $E^{m}\left(u \cdot p^{\alpha}\right)$ there always exist poles which have to be excluded from the contour which go to zero as $p \rightarrow 0$, for example $z=u_{0} p^{\alpha_{0}} q^{k}$ for $k \in \mathbb{Z}_{\geq 0}$. Similarly there are poles going to infinity as $p \rightarrow 0$ which have to be included. The proof of this proposition in essence consists of first picking up the residues belonging to these poles, and taking the contour of the remaining integral close to the unit circle. Subsequently we take the limit as $p \rightarrow 0$ (which involves picking up an increasing number of residues), and show that the sums of these residues converge to one or two basic hypergeometric series, while the remaining integral converges to zero.

Proving that we are allowed to interchange sum and limit and that the remaining integral vanishes in the limit consists of a calculation giving upper bounds on the integrand and residues, after which we can use dominated convergence. This calculation is quite tedious and hence omitted.

The necessary bounds of the elliptic gamma function can be obtained by using the difference equation (2.1) to ensure the argument of the elliptic gamma function is of the form $\Gamma\left(p^{\gamma} z\right)$ for $0 \leq \gamma \leq 1$, and using the known asymptotic behavior of the theta functions outside their poles and zeros. 
This gives a bound on the integrand for a contour which is at least $\epsilon>0$ away from any poles of the integrand, and moreover gives us a summable bound on the residues, thus showing that any residues corresponding to points not of the form $z=t_{0} q^{n}$ must vanish in the limit (here we use $\alpha_{0}<\alpha_{r}$ for $r>0$ ). However a contour as required does in general not exist for all values of $p$.

Therefore choose parameters $u$ in a compact subset $K$ of the complement of the $p$-independent divisors (i.e. such that there are no $p$-independent pole-collisions of the integrand of $E^{m}$ ). For any $p$ for which we can obtain a contour which stays $\epsilon$ away from any poles of the integrand (for all $u \in K$ ), we can use our estimates to bound $\left|E^{m}-B_{\alpha}^{m}\right|$ uniformly for $u \in K$ and $a=|p|$, with the bound going to zero as $a \rightarrow 0$. As long as $\log (p)$ stays $\epsilon$ away from conditions of the form $u_{r}^{-1} u_{s}^{-1} q^{-n}=p^{l+\alpha_{r}+\alpha_{s}}\left(l, n \in \mathbb{N}, u_{r}, u_{s}\right.$ range over the projection of $K$ to the $r$ 'th and $s^{\prime}$ th coordinate) the poles of the integrand near the unit circle stay $\mathcal{O}(\epsilon)$ away from each other and we can find a desired contour. Moreover this ensures that the residues we pick up are at least $\epsilon$ distance away from any other poles.

Note that we only need to consider conditions with $l+\alpha_{r}+\alpha_{s}<0$ as the other condition cannot be satisfied for small enough $p$, this implies there is only a finite set of possible $l, r$ and $s$. Hence, if we start with small enough $K$ and $\epsilon$, we can ensure that these excluded values of $p$ form disjoint sets. In particular we can, in the $p$-plane, create a circle around these disjoint sets, and use the maximum principle to show that $E^{m}-B_{\alpha}^{m}$ is bounded in absolute value inside these circles by the maximum of the absolute value on the circle. As the circle consists entirely of $p$ 's for which our estimates work, we see that inside the circle the difference is bounded as well (by a bound corresponding to a slightly larger radius). Hence for all values of $p$ with $|p|>0$ we find that $\left|E^{m}-B_{\alpha}^{m}\right|$ is bounded uniformly in $u$ and $a=|p|$ with the bound going to zero as $a \rightarrow 0$. in particular the limit holds uniformly for $u \in K$. Finally we can use the Stieltjes-Vitali theorem again to show the limit holds for all values of $u$.

Note that there is some overlap in the conditions of Proposition 4.2 and Proposition 4.3. Indeed we get two different representations of the same function (one integral and one series) in the case of $\alpha \in \mathbb{R}^{2 m+6}$ satisfying $\sum_{r} \alpha_{r}=m+1, \alpha_{0} \leq \alpha_{r} \leq-\alpha_{0}$ for $r=1,2, \alpha_{1}+\alpha_{2}=0$, $-\alpha_{0} \leq \alpha_{r} \leq 1+\alpha_{0}$ for $r \geq 3$.

Moreover, in some special cases we have integral representations of the series in Proposition 4.3, which were not covered in Proposition 4.2. Moreover we sometimes find a second, slightly different, expression for the integrals of Proposition 4.2. Indeed we have

Proposition 4.4. For $\alpha \in \mathbb{R}^{2 m+6}$ satisfying $\sum_{r} \alpha_{r}=m+1$ and $\alpha_{0} \leq \alpha_{1} \leq \cdots \leq \alpha_{2 m+5}$ such that $-1 / 2 \leq \alpha_{0}=\alpha_{1}<0$ and $-\alpha_{0} \leq \alpha_{2}$ and $\alpha_{2 m+5} \leq 1+\alpha_{0}$ the limit in (4.1) exists and we have

$$
\begin{aligned}
B_{\alpha}^{m}(u)= & \prod_{r \geq 2: \alpha_{r}=-\alpha_{0}}\left(u_{0} u_{r}, u_{1} u_{r} ; q\right)(q ; q) \int_{\mathcal{C}} \frac{\theta\left(u_{0} u_{1} w / z, w z ; q\right)}{\theta\left(u_{0} w, u_{1} w ; q\right)} \\
& \times \frac{\prod_{r \geq 2: \alpha_{r}=1+\alpha_{0}}\left(q z / u_{r} ; q\right)}{\prod_{r \geq 2: \alpha_{r}=-\alpha_{0}}\left(u_{r} z ; q\right)} \frac{1}{\left(u_{0} / z, u_{1} / z ; q\right)}\left(\frac{1-z^{2}}{\left(u_{0} z, u_{1} z ; q\right)}\right)^{\left.1_{\left\{\alpha_{0}=-1 / 2\right.}\right\}} \frac{d z}{2 \pi i z}
\end{aligned}
$$

where the contour is a deformation of the unit circle separating the poles in downward sequences from the poles in upward sequences.

The theta functions involving the extra parameter $w$ combine to give a $q$-elliptic function of $w$ and in fact the integrals are independent of $w$ (though this is only obvious from the fact that $B_{\alpha}(u)$ does not depend on $w$ ). In the case $\alpha_{0}=\alpha_{1}=-\alpha_{2}$, which is also treated in Proposition 4.2, we can specialize $w=u_{2}$ to re-obtain the previous integral expression of that limit. 
Proof. As in the proof of Proposition 4.2, we start with the symmetry broken version of $E^{m}$, as in (4.2). Now we specialize $s_{0}=t_{0}, s_{1}=t_{1}$ and $s_{2}=w$. Thus we get

$$
\begin{aligned}
E^{m}(t)= & \frac{\left(p t_{0} t_{1} ; p, q\right)}{\left(q / t_{0} t_{1} ; q\right)} \prod_{r=0,1} \prod_{s=2}^{2 m+5}\left(t_{r} t_{s} ; p, q\right) \prod_{2 \leq r<s \leq 2 m+5}\left(t_{r} t_{s} ; p, q\right)(p ; p)(q ; q) \\
& \times \int_{\mathcal{C}} \prod_{r=0}^{1} \Gamma\left(p t_{r} z, t_{r} / z\right) \prod_{r=2}^{2 m+5} \Gamma\left(t_{r} z^{ \pm 1}\right) \frac{\theta\left(t_{0} t_{1} w / z, w z ; q\right)}{\theta\left(t_{0} w, t_{1} w ; q\right)} \theta\left(z^{-2} ; p\right) \frac{d z}{2 \pi i z} .
\end{aligned}
$$

Replacing $z \rightarrow p^{\alpha_{0}} z$ and $w \rightarrow p^{-\alpha_{0}} w$ and using $t_{r}=p^{\alpha_{r}} u_{r}$ we can subsequently plug in $p=0$ as before to obtain the desired limit.

\section{The polytopes}

In this section we describe a polytope (for each value of $m$ ) such that points of the polytope correspond to vectors $\alpha$ with respect to which we can take limits. Moreover we describe how the limiting functions $B_{\alpha}$ depend on geometrical properties of $\alpha$ in the polytope.

Let us begin by defining the polytopes.

Definition 5.1. For $m \in \mathbb{N}$ we define the vectors $\rho^{(m)}, v_{j_{1} j_{2} \cdots j_{m}}^{(m)}\left(0 \leq j_{1}<j_{2}<\cdots<j_{m} \leq\right.$ $2 m+5)$ and $w_{i j}^{(m)}(0 \leq i<j \leq 2 m+5)$ by

$$
\rho^{(m)}=\frac{1}{2} \sum_{r=0}^{2 m+5} e_{r}, \quad v_{j_{1} j_{2} \cdots j_{m+1}}^{(m)}=\sum_{r=1}^{m+1} e_{j_{r}}, \quad w_{i j}^{(m)}=\rho^{(m)}-e_{i}-e_{j},
$$

where the $e_{k}(0 \leq k \leq 2 m+5)$ form the standard orthonormal basis of $\mathbb{R}^{2 m+6}$. Sometimes we write $v_{S}^{(m)}$ for $S \subset\{0,1, \ldots, 2 m+5\}$ with $|S|=m+1$.

The polytope $P^{(m)}$ is now defined as the convex hull of the vectors $v_{S}^{(m)}(|S|=m+1)$ and $w_{i j}^{(m)}(0 \leq i<j \leq 2 m+5)$. In the notation for both vectors and polytopes we often omit the $(m)$ if the value of $m$ is clear from context.

We will now state the main results of this section. The proofs follow after we have stated all theorems. The main result of this section will be the following theorem.

Theorem 5.2. For $\alpha \in P^{(m)}$ the limit in (4.1) exists and $B_{\alpha}^{m}(u)$ depends only on the (open) face of $P^{(m)}$ which contains $\alpha$, i.e. if $\alpha$ and $\beta$ are contained in the same face of $P^{(m)}$ then $B_{\alpha}^{m}(u)=B_{\beta}^{m}(u)$.

Next we have the following iterated limit property.

Theorem 5.3. Let $\alpha, \beta \in P^{(m)}$. Then the iterated limit property holds, i.e.

$$
\lim _{x \rightarrow 0} B_{\alpha}^{m}\left(x^{\beta-\alpha} u\right)=B_{t \alpha+(1-t) \beta}(u)
$$

for any $0<t<1$.

As $t \alpha+(1-t) \beta$ is contained in the same face of $P^{(m)}$ for all values $0<t<1$, we already know that the right hand side does not depend on $t$.

The iterated limit property shows that all the functions associated to faces can be obtained as limits of the (basic hypergeometric!) functions associated to vertices of the polytope. There are only two different limits associated to vertices (as there are only two different vertices up 
to permutation symmetry), so all results follow from identities satisfied by these two functions. Indeed the idea of this article is not so much to show new identities as it is to show how many known identities fit in a uniform geometrical picture. Moreover this picture allows us to simply classify all formulas of certain kinds.

As an immediate corollary of the iterated limit property we find the last main theorem of this section.

Theorem 5.4. For $\alpha \in P^{(m)}$ the function $B_{\alpha}(u)$ depends only on the space orthogonal to the face containing $\alpha$. To be precise if $\beta$ is in the same (open) face as $\alpha$, then

$$
B_{\alpha}(u)=B_{\alpha}\left(u \cdot x^{\alpha-\beta}\right) .
$$

Proof. Consider the line $v(t)=t \alpha+(1-t) \beta$. As $\alpha$ and $\beta$ are in the same open face there exists $\lambda_{1}>1$ such that $v\left(\lambda_{1}\right)$ is also in this face. Moreover $\alpha$ is a strictly convex linear combination of $v\left(\lambda_{1}\right)$ and $\beta$, and $v\left(\lambda_{1}\right)-\beta=\lambda_{1}(\alpha-\beta)$. Now observe that

$$
B_{\alpha}(u)=\lim _{y \rightarrow 0} B_{v\left(\lambda_{1}\right)}\left(y^{v\left(\lambda_{1}\right)-\beta} u\right)=\lim _{y \rightarrow 0} B_{v\left(\lambda_{1}\right)}\left(y^{v\left(\lambda_{1}\right)-\beta} x^{\frac{v\left(\lambda_{1}\right)-\beta}{\lambda_{1}}} u\right)=B_{\alpha}\left(u \cdot x^{\beta-\alpha}\right)
$$

by the iterated limit property. Here we replaced $y \rightarrow y x^{1 / \lambda_{1}}$ in the second equality.

To prove the first two main theorems, Theorems 5.2 and 5.3 , we need to split up $P^{(m)}$ in several (to be precise $1+(2 m+6)+\left(\begin{array}{c}2 m+6 \\ 3\end{array}\right)$, but essentially only 3 ) different parts. Let us begin with defining the smaller polytopes. Recall the definition of the vectors $\rho^{(m)}, v_{S}^{(m)}$ and $w_{i j}^{(m)}$ from Definition 5.1.

Definition 5.5. We define the three convex polytopes $P_{\mathrm{I}}^{(m)}, P_{\mathrm{II}}^{(m)}$ and $P_{\mathrm{III}}^{(m)}$ by

- $P_{\mathrm{I}}^{(m)}$ is the convex hull of the vectors $v_{S}^{(m)}(S \subset\{0,1, \ldots, 2 m+5\})$;

- $P_{\mathrm{II}}^{(m)}$ is the convex hull of the vectors $v_{S}^{(m)}(S \subset\{1,2, \ldots, 2 m+5\})$ and $w_{0 j}^{(m)}(1 \leq j \leq$ $2 m+5)$;

- $P_{\mathrm{III}}^{(m)}$ is the convex hull of the vectors $v_{S}^{(m)}(S \subset\{3,4, \ldots, 2 m+5\})$ and $w_{i j}^{(m)}(0 \leq i<j \leq 2)$.

Here we always have $|S|=m+1$ (otherwise $v_{S}^{(m)}$ would not make sense).

The polytopes $P_{\mathrm{I}}^{(m)}, P_{\mathrm{II}}^{(m)}$ and $P_{\mathrm{III}}^{(m)}$ correspond to limits in Propositions 4.1, 4.3, respectively 4.2. The following proposition allows us to prove things about $P^{(m)}$ by proving them for these simpler polytopes.

Proposition 5.6. Denote $\sigma(A)=\{\sigma(a) \mid a \in A\}$ for some permutation $\sigma \in S_{2 m+6}$. Then we have

$$
P^{(m)}=P_{\mathrm{I}}^{(m)} \cup \bigcup_{\sigma \in S_{2 m+6}} \sigma\left(P_{\mathrm{II}}^{(m)}\right) \cup \bigcup_{\sigma \in S_{2 m+6}} \sigma\left(P_{\mathrm{III}}^{(m)}\right) .
$$

Proof. It is sufficient to show that given any set $V$ of vertices of $P^{(m)}$ their convex hull can be written as the union of subsets of the polytopes on the right hand side. If $V$ does not contain one of the following bad sets

1. $\left\{w_{i j}, v_{S_{1}}, v_{S_{2}}\right\}$ for $i \in S_{1}, j \in S_{2}$;

2. $\left\{w_{i j}, v_{S}\right\}$ for $i, j \in S$;

3. $\left\{w_{i j}, w_{k l}\right\}$; 


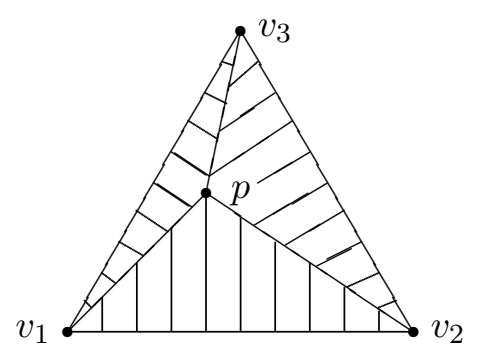

Figure 1. $\operatorname{ch}\left(v_{1}, v_{2}, v_{3}\right)=\operatorname{ch}\left(v_{1}, v_{2}, p\right) \cup \operatorname{ch}\left(v_{1}, v_{3}, p\right) \cup \operatorname{ch}\left(v_{2}, v_{3}, p\right)$.

4. $\left\{w_{i j}, w_{i k}, v_{S}\right\}$ for $i \in S$,

where $i, j, k, l$ denote different integers, then $V$ is contained in the sets of vertices of $P_{\mathrm{I}}^{(m)}$ or one of the permutations of $P_{\text {II }}$ or $P_{\text {III }}$. This follows from a simple case analysis depending on the number and kind of $w_{i j}$ 's in $V$.

Given any point $p$ in the (closed) convex hull $\operatorname{ch}(V)$ of $V$, with $p=\sum_{v \in V} a_{v} v$, we can write $\operatorname{ch}(V)=\bigcup_{v: a_{v}>0} \operatorname{ch}((V \backslash\{v\}) \cup\{p\})$. Indeed any point $q$ in $\operatorname{ch}(V)$ can be written as $q=\sum_{v \in V} b_{v} v=\gamma p+\sum_{v \in V}\left(b_{v}-a_{v} \gamma\right) v$, where we can take $\gamma \geq 0$ to be such that $b_{v^{\prime}}=a_{v^{\prime}} \gamma$ for some $v^{\prime}$ with $a_{v^{\prime}}>0$ and $b_{v} \geq a_{v} \gamma$ for all $v \in V$. Now $q$ clearly is a convex linear combination of elements of $\left(V \backslash\left\{v^{\prime}\right\}\right) \cup\{p\}$. This argument is visualized in Fig. 1. As a generalization we obtain that if $p \in \operatorname{ch}(W)$ for some set $W$ we have that $\operatorname{ch}(V) \subset \bigcup_{v: a_{v}>0} \operatorname{ch}((V \backslash\{v\}) \cup W)$.

Now we can consider a set of vertices $V$ containing a bad configuration, and use the above method to rewrite $\operatorname{ch}(V) \subset \bigcup_{i} \operatorname{ch}\left(V_{i}\right)$, where the $V_{i}$ are sets of vertices of $P^{(m)}$ that do not contain that bad configuration, while not introducing any new bad configurations. Iterating this we end up with $\operatorname{ch}(V) \subset \bigcup_{i} \operatorname{ch}\left(V_{i}\right)$ for some sets $V_{i}$ without bad configurations; in particular $\operatorname{ch}(V)$ is contained in the right hand side of (5.1).

First we consider a bad set of the form $\left\{w_{i j}, w_{k l}\right\}$. Then $p=\frac{1}{2}\left(w_{i j}+w_{k l}\right)=\frac{1}{2}\left(v_{T_{1}}+v_{T_{2}}\right)$, where $T_{1}$ and $T_{2}$ are any two sets of size $\left|T_{i}\right|=m+1$ with $T_{1} \cup T_{2} \cup\{i, j, k, l\}=\{0,1, \ldots, 2 m+5\}$. Thus we get $V_{1}=\left(V \cup\left\{v_{T_{1}}, v_{T_{2}}\right\}\right) \backslash\left\{w_{i j}\right\}$ and $V_{2}=\left(V \cup\left\{v_{T_{1}}, v_{T_{2}}\right\}\right) \backslash\left\{w_{k l}\right\}$, as new sets. In particular the number of $w$ 's decreases and we can iterate this until no bad sets of the form $\left\{w_{i j}, w_{k l}\right\}$ exist.

For the remaining three bad kind of sets we just indicate the way a strictly convex combination of the vectors in the bad set can be written in terms of better vectors. In each step we assume there are no bad sets of the previous form, to ensure we do not create any new bad sets (at least not of the form currently under consideration or of a form previously considered).

1. For $\left\{w_{i j}, v_{S}\right\}$ with $i, j \in S$ we have $\frac{2}{3} w_{i j}+\frac{1}{3} v_{S}=\frac{1}{3}\left(v_{T_{1}}+v_{T_{2}}+v_{U}\right)$ where $S \backslash T_{1}=S \backslash T_{2}=$ $\{i, j\}$ and $S \cap U=T_{1} \cap U=T_{2} \cap U=\varnothing$ and $T_{1} \cap T_{2}=S \backslash\{i, j\}$ (thus $T_{1}, T_{2}$ and $U$ cover all the elements of $S$, except $i$ and $j$, twice, and all other points once).

2. For $\left\{w_{i j}, w_{i k}, v_{S}\right\}$ with $i \in S$ we have $\frac{1}{3}\left(w_{i j}+w_{i k}+v_{S}\right)=\frac{1}{3}\left(v_{T_{1}}+v_{T_{2}}+v_{U}\right)$ for $S \backslash T_{1}=$ $S \backslash T_{2}=\{i\}$ and $S \cap U=T_{1} \cap U=T_{2} \cap U=\varnothing$ and $T_{1} \cap T_{2}=S \backslash\{i\}$ and $j, k \notin T_{1}, T_{2}, U$.

3. For $\left\{w_{i j}, w_{i k}, v_{S}\right\} \subset V$, with $i \in S$, then $j, k \notin S$ and $\frac{1}{3}\left(w_{i j}+w_{i k}+v_{S}\right)=\frac{1}{3}\left(v_{T_{1}}+v_{T_{2}}+v_{U}\right)$ for $S \backslash T_{1}=S \backslash T_{2}=\{i\}$ and $S \cap U=T_{1} \cap U=T_{2} \cap U=\varnothing$ and $T_{1} \cap T_{2}=S \backslash\{i\}$ and $j, k \notin T_{1}, T_{2}, U$.

Let us now consider the bounding inequalities related to these polytopes.

Proposition 5.7. The polytopes $P^{(m)}, P_{\mathrm{I}}^{(m)}, P_{\mathrm{II}}^{(m)}$ and $P_{\mathrm{III}}^{(m)}$ are the subsets of the hyperplane $\left\{\alpha: \alpha \in \mathbb{R}^{2 m+6} \mid \sum_{i} \alpha_{i}=m+1\right\}$ described by the following bounding inequalities 
- For $P^{(m)}$ the bounding inequalities are

$$
\begin{array}{ll}
-\frac{1}{2} \leq \alpha_{i} \leq 1, & (0 \leq i \leq 2 m+5), \\
\alpha_{i} \leq 1+\alpha_{j}+\alpha_{k}+\alpha_{l}, & (|\{i, j, k, l\}|=4), \\
\alpha_{i}-\alpha_{j} \leq 1, & (i \neq j), \\
(|S|-2) \alpha_{i}+\sum_{j \in S} \alpha_{j} \geq 0, & (i \notin S, 3 \leq|S| \leq m+3) .
\end{array}
$$

For $m=0$ the equations $\alpha_{r} \leq 1$ and $\alpha_{i} \leq 1+\alpha_{j}+\alpha_{k}+\alpha_{l}$ are valid but not bounding.

- The polytope $P_{\mathrm{I}}^{(m)}$ is described by the bounding inequalities

$$
0 \leq \alpha_{i} \leq 1, \quad(0 \leq i \leq 2 m+5) .
$$

For this polytope too, if $m=0$ the equations $\alpha_{r} \leq 1$ are valid but not bounding.

- The polytope $P_{\mathrm{II}}^{(m)}$ is described by the bounding inequalities

$$
\begin{array}{ll}
-1 / 2 \leq \alpha_{0}, & \\
\alpha_{r}-\alpha_{0} \leq 1, & (r \geq 1), \\
(|S|-2) \alpha_{0}+\sum_{j \in S} \alpha_{j} \geq 0, & (0 \notin S, 0 \leq|S| \leq m+3) .
\end{array}
$$

- Finally, the polytope $P_{\mathrm{III}}^{(m)}$ is described by the bounding inequalities

$$
\begin{array}{ll}
\alpha_{i}+\alpha_{j} \leq 0, & (0 \leq i<j \leq 2), \\
-\alpha_{i} \leq \alpha_{0}+\alpha_{1}+\alpha_{2}, & (3 \leq i \leq 2 m+5), \\
\alpha_{i}-1 \leq \alpha_{0}+\alpha_{1}+\alpha_{2}, & (3 \leq i \leq 2 m+5) .
\end{array}
$$

If $m=0$ the equations $\alpha_{i}-1 \leq \alpha_{0}+\alpha_{1}+\alpha_{2}$ are valid but not bounding.

Proof. It can be immediately verified that the vertices of the polytopes $P^{(m)}, P_{\mathrm{I}}^{(m)}, P_{\mathrm{II}}^{(m)}$ and $P_{\text {III }}^{(m)}$ satisfy the relevant inequalities, hence so does any convex linear combination of them. In particular it is clear that the polytopes are contained in the sets defined by these bounding inequalities.

Note that the different polytopes have symmetries of $S_{2 m+6}$ (for $P^{(m)}$ and $P_{\mathrm{I}}^{(m)}$ ), respectively $S_{1} \times S_{2 m+5}\left(P_{\text {II }}^{(m)}\right)$, respectively $S_{3} \times S_{2 m+3}\left(P_{\text {III }}^{(m)}\right)$. We only have to find the bounding inequalities of these polytopes intersected with a Weyl chamber of the relevant symmetry group, as all bounding inequalities will be permutations of these. These bounding inequalities can be written in the form $\mu \cdot \alpha \geq 0$ for each $\alpha$ in the polytope; we do not need affine equations as we have $\sum_{r} \alpha_{r}=m+1$.

A bounding inequality must attain equality at a codimension 1 space of the vertices of the polytope; in particular if we consider all subsets $V$ of $2 m+5$ vertices of the intersection of each polytope with the relevant Weyl chamber and insist on $\mu \cdot v=0$ for each $v \in V$, we find all bounding inequalities (and perhaps some more inequalities). For $P_{\mathrm{I}}, P_{\mathrm{II}}$ and $P_{\mathrm{III}}$ we are in the circumstance that there are $2 m+6$ vertices for the intersection of the Weyl chamber with the polytope; in particular each set of $2 m+5$ vertices corresponds to leaving one vector out. Moreover the sign of $\mu$ is then determined by insisting on $\mu \cdot v>0$ for the remaining vertex $v$. As the equations are all homogeneous the normalization of $\mu$ is irrelevant.

Let us consider the case of $P_{\mathrm{II}}$. The set of relevant vertices is $\left\{v_{S}, w_{01}, e_{1}-e_{2}, \ldots, e_{2 m+4}-\right.$ $\left.e_{2 m+5}\right\}$ for $S=\{m+6, \ldots, 2 m+5\}$. We now have the following options for leaving one vector out. 
1. If $\mu \cdot v_{S}^{(m)}>0$ we get $\mu=\rho+(m+1) e_{0}$, thus the equation $\alpha_{0} \geq-1 / 2$.

2. If $\mu \cdot w_{01}^{(m)}>0$ we get $\mu=-e_{0}$ and the equation $\alpha_{0} \leq 0$.

3. If $\mu \cdot\left(e_{i}-e_{i+1}\right)>0$ for $1 \leq i \leq m+3$ we get $\mu=(i-2) e_{0}+\sum_{r=1}^{i} e_{r}$ and the equation becomes $(i-2) \alpha_{0}+\sum_{r=1}^{i} e_{r} \geq 0$.

4. If $\mu \cdot e_{i}-e_{i+1}>0$ for $m+4 \leq i \leq 2 m+4$ we get $\mu=(m+2)(2 m+5-i) e_{0}+(2 m+$ $5-i) \sum_{r=1}^{i} e_{r}+(m+4-i) \sum_{r=i+1}^{2 m+5} e_{r}$ and the equation becomes $\left(\alpha_{0}+1\right)(2 m+5-i) \geq$ $\sum_{r=i+1}^{2 m+5} \alpha_{r}$.

Note that the equation $\alpha_{0} \leq 0$ is the $|S|=0$ case of $(|S|-2) \alpha_{0}+\sum_{j \in S} \alpha_{j} \geq 0$. Now the last set of equations all follow from the instance $i=2 m+4$, i.e. $\alpha_{0}+1 \geq \alpha_{2 m+5}$ and the equation $\alpha_{2 m+5} \geq \alpha_{r}$. The rest are true bounding inequalities. It is only hard to see that the solutions to $(i-2) \alpha_{0}+\sum_{r=1}^{i} e_{r}=0$ in the set of vertices of the polytope span a codimension one space; however the set $\left\{w_{01}, \ldots, w_{0 i}\right\} \cup\left\{v_{T} \mid T \subset\{i+1, \ldots, 2 m+5\}\right\}$ does span a set of codimension one.

In a similar way one obtains the bounding inequalities for $P_{\mathrm{I}}$ and $P_{\mathrm{III}}$, we omit the explicit calculations here. To obtain the bounding inequalities of $P$ itself, we observe that any bounding inequality of $P$ must be a bounding inequality of one of $P_{\mathrm{I}}, P_{\mathrm{II}}, P_{\mathrm{III}}$ or one of their permutations, as $P$ is the union of those polytopes. Indeed any of these equations which are valid on $P$ are bounding inequalities (as the span of the set of vertices for which equality holds does not reduce in dimension when going from a smaller polytope to $P$ ). Thus we can find the bounding inequalities for $P$ by checking which of the bounding inequalities of these smaller polytopes are valid on $P$. This we only need to check on the vertices of $P$, which is a straightforward calculation.

Note that we could also have obtained the bounding inequalities for $P$ in the same way that we obtained those of $P_{\mathrm{I}}, P_{\mathrm{II}}$ and $P_{\mathrm{III}}$. However now we would have to take $2 m+5$ vectors from the set $\left\{v_{S}, w_{01}, e_{0}-e_{1}, \ldots, e_{2 m+4}-e_{2 m+5}\right\}$, which has $2 m+7$ elements. The number of options therefore becomes quite large, thus we prefer to avoid this method.

We would like to give special attention to the bounding inequalities of $P^{(1)}$, which is the polytope which interests us most. We can rewrite these bounding inequalities in a clearly $W\left(E_{7}\right)$ invariant way.

Proposition 5.8. The bounding inequalities for $P^{(1)}$ inside the subspace $\alpha \cdot \rho=1$ are given by

$$
\begin{array}{ll}
\alpha \cdot \delta \leq 1, & \left(\delta \in R\left(E_{7}\right)\right), \\
\alpha \cdot \mu \leq 2, & \left(\mu \in \Lambda\left(E_{8}\right), \mu \cdot \rho=1, \mu \cdot \mu=4\right)
\end{array}
$$

for $\alpha \in P^{(1)}$. Here $\Lambda\left(E_{8}\right)=\mathbb{Z}^{8} \cup\left(\mathbb{Z}^{8}+\rho\right)$ is the root lattice of $E_{8}$.

Proof. Up to $S_{8}$ one can classify the roots of $E_{7}$, giving $\delta=e_{i}-e_{j}$ or $\delta=\rho-e_{i}-e_{j}-e_{k}-e_{l}$, which handles the bounding inequalities $\alpha_{i}-\alpha_{j} \leq 1$ and $\alpha_{i}+\alpha_{j}+\alpha_{k}+\alpha_{l} \leq 0$. Similarly we can classify all relevant $\mu \in \Lambda\left(E_{8}\right)$ as $\mu=2 e_{i}, \mu=e_{i}+e_{j}+e_{k}-e_{l}, \mu=\rho-2 e_{i}$ and $\mu=\rho+e_{i}-e_{j}-e_{k}-e_{l}$, the corresponding equations are again directly related to the bounding inequalities of $P^{(1)}$ as given in Proposition 5.7.

It is convenient to rewrite the integral limits of Propositions 4.1 and 4.2 in a uniform way which clearly indicates the bounding inequalities for the corresponding polytopes (i.e. $P_{\mathrm{I}}$, resp. $P_{\mathrm{III}}$ ). It is much harder to give such a uniform expression for $P_{\mathrm{II}}$ (and we need separate expressions for the intersection with $P_{\mathrm{I}}$ and $P_{\mathrm{III}}$ and the facet $\left.\left\{\alpha_{0}=1+\alpha_{2 m+5}\right\}\right)$, so we omit those. 
Proposition 5.9. Define vectors $v_{j}=e_{j}$ and $w_{j}=e_{j}-\frac{2}{m+1} \rho$, then the bounding inequalities for $P_{\mathrm{I}}^{(m)}$ become $v_{j} \cdot \alpha \geq 0, w_{j} \cdot \alpha \geq 0$ (and the condition $2 \rho \cdot \alpha=m+1$ ). The limit can be written as

$$
B_{\alpha}^{m}(u)=\prod_{j \neq k: v_{j} \cdot \alpha=v_{k} \cdot \alpha=0}\left(u^{v_{j}+v_{k}} ; q\right) \frac{(q ; q)}{2} \int \frac{\left(z^{ \pm 2} ; q\right) \prod_{j: w_{j} \cdot \alpha=0}\left(u^{w_{j}} z^{ \pm 1} ; q\right)}{\prod_{j: v_{j} \cdot \alpha=0}\left(u^{v_{j}} z^{ \pm 1} ; q\right)} \frac{d z}{2 \pi i z} .
$$

Proposition 5.10. Define the vectors $v_{j}=e_{0}+e_{1}+e_{2}-e_{j}(0 \leq j \leq 2), w_{j}=e_{0}+e_{1}+e_{2}+e_{j}$ $(3 \leq j \leq 2 m+5)$, and $x_{j}=e_{0}+e_{1}+e_{2}-e_{j}-\frac{2}{m+1} \rho(3 \leq j \leq 2 m+5)$, then the bounding inequalities for $P_{\mathrm{III}}^{(m)}$ can be written as $v_{j} \cdot \alpha \geq 0, w_{j} \cdot \alpha \geq 0$ and $x_{j} \cdot \alpha \geq 0$ (together with $2 \rho \cdot \alpha=m+1)$. Let $y=w_{j}+x_{j}$ (note $y$ is independent of $j$ ) then

$$
\begin{aligned}
B_{\alpha}^{m}(u)= & \frac{\prod_{j: w_{j} \cdot \alpha=0} \prod_{k: v_{k} \cdot \alpha=0}\left(u^{w_{j}+u_{k}} ; q\right)}{\prod_{k: v_{k} \cdot \alpha=0}\left(q u^{v_{k}} ; q\right)}\left(\prod_{r \neq s: v_{r} \cdot \alpha=v_{s} \cdot \alpha=0}\left(q u^{y+v_{r}+v_{s}} ; q\right)\right)^{1_{\{y \cdot \alpha=0\}}} \\
& \times \int \theta(1 / z ; q) \frac{\prod_{j: x_{j} \cdot \alpha=0}\left(q^{2} z u^{x_{j}} ; q\right)}{\prod_{j: w_{j} \cdot \alpha=0}\left(z u^{w_{j}} ; q\right)} \frac{\prod_{r \neq s: v_{r} \cdot \alpha=v_{s} \cdot \alpha=0}\left(q u^{v_{r}+v_{s}} / z ; q\right)}{\prod_{r: v_{r} \cdot \alpha=0}\left(u^{\left.v_{r} / z ; q\right)}\right.} \\
& \times\left(\frac{\left(1-q u^{y} z^{2}\right) \prod_{r \neq s: v_{r} \cdot \alpha=v_{s} \cdot \alpha=0}\left(q^{2} z u^{y+v_{r}+v_{s}} ; q\right)}{\prod_{r: v_{r} \cdot \alpha=0}\left(q z u^{y+v_{r}} ; q\right)}\right)^{1_{\{y \cdot \alpha=0\}}} \frac{d z}{2 \pi i z} .
\end{aligned}
$$

Proof. These two propositions are just a rewriting of Propositions 4.1 and 4.2.

With these expressions the proof of the following proposition becomes fairly straightforward.

Proposition 5.11. Let the polytope $Q$ be either $P_{\mathrm{I}}^{(m)}, P_{\mathrm{II}}^{(m)}$ or $P_{\mathrm{III}}^{(m)}$. For $\alpha \in Q$ the limit in (4.1) exists and depends only on the face of $Q$ which contains $\alpha$ (i.e. if $\alpha$ and $\beta$ are contained in the same face of $Q$ then $\left.B_{\alpha}^{m}(u)=B_{\beta}^{m}(u)\right)$.

Proof. Indeed Propositions 5.9, respectively 5.10 give the limits for the vectors $\alpha$ in $P_{\mathrm{I}}^{(m)}$, respectively $P_{\text {III }}^{(m)}$. For $P_{\text {II }}^{(m)}$ the limits are given in Proposition 4.3, except for the cases with $\alpha_{0}=0$ (which is the intersection with $P_{\mathrm{I}}^{(m)}$ ), and $\alpha_{1}+\alpha_{2}=0$ (the intersection with $P_{\mathrm{III}}^{(m)}$ ), or a permutation of such a case. In particular we have obtained limits in those cases as well.

For $P_{\mathrm{I}}$ and $P_{\mathrm{III}}$ the expressions in the previous two propositions immediately show that the limits only depend on which bounding inequalities are strict or not, and hence on the face of the polytope containing $\alpha$. For $P_{\text {II }}$ we note that the conditions $\alpha_{0}=0$ and $\alpha_{1}+$ $\alpha_{2}=0$ (governing which proposition to look at) correspond to bounding inequalities. Within Proposition 4.3 we observe that the condition $\alpha_{r}=-\alpha_{0}$ becomes a bounding equation once $2 \alpha_{0}=\sum_{r \geq 1: \alpha_{0}+\alpha_{r}<0} \alpha_{r}+\alpha_{0}$ holds (as the difference of the equations with $r \in S$ and with $r \notin S)$. So also for $P_{\mathrm{II}}$ the limit only depends on which bounding inequalities are strict and which not.

We can now prove the first of the main theorems, the equivalent result for the full polytope $P^{(m)}$.

Proof of Theorem 5.2. By the $S_{2 m+6}$ symmetry of $E_{m}(t)$ we see that if a limit exists for some $\alpha$, then it also exists for all permutations of $\alpha$. As $P^{(m)}$ is the union of permutations 
of $P_{\mathrm{I}}^{(m)}, P_{\mathrm{II}}^{(m)}$ and $P_{\mathrm{III}}^{(m)}$, by the previous proposition we find that the limit $B_{\alpha}^{m}$ exists for all $\alpha \in P^{(m)}$. We would like to extend the statement about dependence on faces as well. To prove this it would be sufficient to show that all faces of $P^{(m)}$ are in fact a face of one of the polytopes in its decomposition, however this is not true. We do have the following lemma.

Lemma 5.12. All faces of $P^{(m)}$ are faces of either $P_{\mathrm{I}}^{(m)}, \sigma\left(P_{\mathrm{II}}^{(m)}\right)$ or $\sigma\left(P_{\mathrm{III}}^{(m)}\right)$ for some $\sigma \in$ $S_{2 m+6}$, except the interior of $P^{(m)}$ and permutations of the facet given by the equality $\alpha_{0}+\alpha_{1}+$ $\alpha_{2}+\alpha_{3}=0$.

Proof. Any face of $P^{(m)}$ can be written as the set of all convex linear combinations of some set $V$ of vertices of $P^{(m)}$. Recall that $V$ is contained in the set of vertices of $P_{\mathrm{I}}, P_{\mathrm{II}}$ or $P_{\mathrm{III}}$ (or a permutation thereof), unless it contains one of the four bad sets in the proof of Proposition 5.6. Therefore, except when $V$ contains bad sets, the face determined by $V$ is a face of $P_{\mathrm{I}}, P_{\mathrm{II}}$ or $P_{\mathrm{III}}$. We now show that if $V$ contains a bad set, the convex hull of $V$ contains a point in the interior of $P^{(m)}$ or the interior of the facet given by $\alpha_{0}+\alpha_{1}+\alpha_{2}+\alpha_{3}=0$. Hence $\operatorname{ch}(V)$ is either equal to the interior of $P^{(m)}$, or to the special facet.

1. If $\left\{w_{i j}, v_{S_{1}}, v_{S_{2}}\right\} \subset V\left(i \in S_{1}, j \in S_{2}\right)$ then $\left(w_{i j}+v_{S_{1}}+v_{S_{2}}\right) / 3 \in \operatorname{ch}(V)$. This is a point where all elements are $1 / 6,1 / 2$ or $5 / 6$, in particular it is a point in the interior of $P_{\mathrm{I}}$, and thus of $P$ itself.

2. If $\left\{w_{i j}, v_{S}\right\} \subset V(i, j \in S)$, then $\left(w_{i j}+v_{S}\right) / 2 \in \operatorname{ch}(V)$, which is in the interior of $P_{\mathrm{I}}$.

3. If $\left\{w_{i j}, w_{i k}, v_{S}\right\} \subset V,(i \in S)$, then $\left(w_{i j}+w_{i k}+2 v_{S}\right) / 4 \in \operatorname{ch}(V)$, which is again a point in the interior of $P_{\mathrm{I}}$.

4. If $\left\{w_{i j}, w_{k l}\right\} \subset V$, then $\left(w_{i j}+w_{k l}\right) / 2 \in \operatorname{ch}(V)$. All bounding inequalities of $P$ are strict on this point except $\alpha_{i}+\alpha_{j}+\alpha_{k}+\alpha_{l}=0$, thus it is a point in the interior of the corresponding facet. Thus $\operatorname{ch}(V)$ is either this facet or the interior of $P$.

Now it remains to show that the function $B_{\alpha}^{m}$ is the same for all points in the interior, and all points on the facet given by $\alpha_{0}+\alpha_{1}+\alpha_{2}+\alpha_{3}=0$. This follows from the following lemma.

Lemma 5.13. On the facet of $P^{(m)}$ given by $\alpha_{0}+\alpha_{1}+\alpha_{2}+\alpha_{3}=0$ we have $B_{\alpha}^{m}(u)=$ $\left(u_{0} u_{1} u_{2} u_{3} ; q\right)$. Moreover on the interior of $P^{(m)}$ we have $B_{\alpha}^{m}(u)=1$.

Proof. In the $m=0$ case we find that the right hand side of the evaluation formula (3.2) converges for $\alpha$ on the facet to $\left(q / u_{4} u_{5} ; q\right)=\left(u_{0} u_{1} u_{2} u_{3} ; q\right)$, while in the interior of $P^{(m)}$ the limit converges to 1 (as for $m=0$ the condition $\alpha_{r}+\alpha_{s}=1$ is equivalent to the sum of the other four parameters being zero.). Thus for $m=0$ the lemma is true.

For $m>0$ we can classify all the faces of $P_{\mathrm{I}}^{(m)}, P_{\mathrm{II}}^{(m)}$ and $P_{\mathrm{III}}^{(m)}$ which intersect the given facet and the interior of $P^{(m)}$. The bounding inequality $\alpha_{0}+\alpha_{1}+\alpha_{2}+\alpha_{3} \geq 0$ implies that the only vertices allowed in the closure of this facets are $v_{S}$ for $0,1,2,3 \notin S$ and $w_{i j}$ for $i, j \in\{0,1,2,3\}$. We obtain the following set of faces of $P_{\mathrm{I}}, P_{\mathrm{II}}$ and $P_{\mathrm{III}}$ in the facet $\alpha_{0}+\alpha_{1}+\alpha_{2}+\alpha_{3}=0$ modulo permutations of the parameters.

\begin{tabular}{l|l|l} 
Polytope & Vertices & Relations \\
\hline$P_{\mathrm{I}} \cap P_{\mathrm{II}} \cap P_{\mathrm{III}}$ & $v_{S}(0,1,2,3 \notin S)$ & $\alpha_{0}=\alpha_{1}=\alpha_{2}=\alpha_{3}=0$ \\
$P_{\mathrm{II}} \cap P_{\mathrm{III}}$ & $w_{01}, v_{S}(0,1,2,3 \notin S)$ & $\alpha_{0}=\alpha_{1}=-\alpha_{2}=-\alpha_{3}$ \\
& $w_{01}, w_{02}, v_{S}(0,1,2,3 \notin S)$ & $\alpha_{0}+\alpha_{3}=0, \alpha_{1}=\alpha_{2}=0$ \\
$P_{\mathrm{II}}$ & $w_{01}, w_{02}, w_{03}, v_{S}(0,1,2,3 \notin S)$ & $\alpha_{0}<\alpha_{r}<-\alpha_{0}(r=1,2,3)$ \\
$P_{\mathrm{III}}$ & $w_{01}, w_{02}, w_{12}, v_{S}(0,1,2,3 \notin S)$ & $-\alpha_{3}<\alpha_{r}<\alpha_{3}(r=0,1,2)$
\end{tabular}


We omitted the conditions on $\alpha_{r}$ for $r \geq 4$ as they are the same in each case. Indeed the bounding inequalities imply that $-\beta<\alpha_{r}<1+\beta$ for $r \geq 4$, where $\beta$ is the sum of the three smallest parameters. The classification becomes apparent once we realize that the $v_{S}$ part of the vertices of the faces is fixed (they must contain a point $v_{S}$ with $0,1,2,3 \notin S$ to be in the open facet, while they cannot contain any other $v_{S}$ ). Thus we only have to consider the possibilities for adding some $w_{i j}$ 's. In these five faces we can directly check what the limit is, and observe that the corresponding integrals and sums indeed evaluate to the desired $\left(u_{0} u_{1} u_{2} u_{3} ; q\right)$. The required evaluation identities are provided by the $m=0$ cases of these faces.

Similarly we can describe all faces of $P_{\mathrm{I}}, P_{\mathrm{II}}$ and $P_{\mathrm{III}}$ (modulo permutations) meeting the interior of $P$.

\begin{tabular}{l|l|l} 
Polytope & Vertices & Relations \\
\hline$P_{\mathrm{I}} \cap P_{\mathrm{II}} \cap P_{\mathrm{III}}$ & $v_{S},(0,1,2 \notin S)$ & $\alpha_{0}=\alpha_{1}=\alpha_{2}=0$ \\
$P_{\mathrm{I}} \cap P_{\mathrm{II}}$ & $v_{S}(0,1 \notin S)$ & $\alpha_{0}=\alpha_{1}=0<\alpha_{2}<1$ \\
& $v_{S}(0 \notin S)$ & $\alpha_{0}=0<\alpha_{1}, \alpha_{2}<1$ \\
$P_{\mathrm{I}}$ & $v_{S}$ & $0<\alpha_{0}, \alpha_{1}, \alpha_{2}<1$ \\
$P_{\mathrm{II}} \cap P_{\mathrm{III}}$ & $w_{01}, v_{S}(0,1,2 \notin S)$ & $-1 / 2<\alpha_{0}=\alpha_{1}=-\alpha_{2}$ \\
& $w_{01}, w_{02}, v_{S}(0,1,2 \notin S)$ & $-1 / 2<\alpha_{0}<\alpha_{1}=-\alpha_{2}$ \\
$P_{\mathrm{III}}$ & $w_{01}, w_{02}, w_{12}, v_{S}(0,1,2 \notin S)$ & $\alpha_{r}+\alpha_{s}<0,(r, s \in\{0,1,2\})$ \\
$P_{\mathrm{II}}$ & $w_{01}, v_{S}(0,1, \notin S)$ & $-1 / 2<\alpha_{0}=\alpha_{1}>-\alpha_{2}$ \\
& $w_{01}, w_{02}, w_{03}, v_{S}(0 \notin S)$ & $\alpha_{0}<0$ and $\alpha_{0}<\alpha_{1}>-\alpha_{2}$
\end{tabular}

For each face we have the extra conditions $-\beta<\alpha_{r}<1+\beta$ for $r \geq 3$. We can check for all these 9 faces that the value on that face equals 1 identically. Again the required evaluations all follow from the $m=0$ case.

We conclude that also on the two types of faces of $P^{(m)}$ which are not a face of one of the subpolytopes, the value is the same on the entire face.

We can now also prove the second main theorem, the iterated limit property.

Proof of Theorem 5.3. It is sufficient to prove this property for the closed polytopes $P_{\mathrm{I}}^{(m)}$, $P_{\mathrm{II}}^{(m)}$ and $P_{\mathrm{III}}^{(m)}$. Observe that for the vector $t \alpha+(1-t) \beta$ precisely those boundary conditions (of the respective polytopes) are strict which are strict for either $\alpha$ or $\beta$.

In Propositions 5.9 and 5.10 we have written the limits in $P_{\mathrm{I}}$ and $P_{\mathrm{III}}$ as an integral of a product of terms $f(u, w)=\left(u^{w} ; q\right)^{1_{\{w \cdot \alpha=0\}}}$ where $w \cdot \alpha \geq 0$ is the sum of some bounding inequalities. In particular in the expression for $B_{t \alpha+(1-t) \beta}^{m}$ only those terms remain corresponding to sums of bounding inequalities which are attained in both $\alpha$ and $\beta$. On the other hand, as $\left(x^{\beta-\alpha} u\right)^{w}=x^{(\beta-\alpha) \cdot w} u^{w}$ we find that if $w \cdot \alpha=w \cdot \beta=0$ then the term $f_{\alpha}\left(x^{\beta-\alpha} u, w\right)$ is constant in $x$ and thus does not change in the limit, while if $w \cdot \alpha>0$, we find that $f_{\alpha}\left(x^{\beta-\alpha} u, w\right)=1$ is again independent of $x$, and finally if $w \cdot \alpha=0<w \cdot \beta$, then we have a uniform limit $\lim _{x \rightarrow 0} f_{\alpha}\left(x^{\beta-\alpha} u, w\right)=\lim _{x \rightarrow 0}\left(x^{\beta \cdot w} u^{w} ; q\right)=1=f_{t \alpha+(1-t) \beta}(u, w)$. Thus to prove iterated limits we only have to show we are allowed to interchange limit and integral, which follows from the fact that we integrate over some compact contour, and the fact that the $x$-dependent poles which have to remain inside the contour converge to 0 , while the $x$-dependent poles which have to remain outside the contour go to infinity; in particular for $x$ small enough we can take an $x$-independent contour.

To prove the result for $P_{\mathrm{II}}$ is more complicated as we do not have a uniform description of the limit. For the closed facets determined by $\alpha_{0}=0, \alpha_{1}+\alpha_{2}=0$ or $\alpha_{0}=\alpha_{1}$ we have an integral description (see Propositions 4.1, 4.2 and 4.4), and limits within these facets can be treated as for $P_{\mathrm{I}}$ and $P_{\mathrm{III}}$. For the complement of these facets the limit is given in Proposition 4.3 as a single sum (possibly of only one term), and we can replicate the argument for $P_{\mathrm{I}}$ and $P_{\mathrm{III}}$ for 
sums instead of integrals to see that iterated limits within the complement of the facets hold. We are left with showing that limits from one of the three facets to the inside behave correctly.

These limits all pass from an integral to a single sum. The simplest argument we found is to simply calculate these limits in all three cases separately (due to the rather uniform expressions of the integrals and single sums, we can handle the different faces in each of the closed facets uniformly). It boils down to picking the residues associated with poles which either go to zero as $x \rightarrow 0$, while they should be outside the contour, or go to infinity while they should be inside the contour. Subsequently we bound the integrand around the unit circle to show that the remaining integral vanishes in the limit. Finally we give a bound on the residues and use dominated convergence to show we are allowed to interchange limit and sum. This bound also serves to show that the sum of the residues which are not associated to $u_{0}$ (where we take the first coefficient in $\alpha$ to be the strictly lowest one) vanishes as well. The calculations involved are again tedious, and very similar to the calculations in the proof of Proposition 4.3.

\section{Transformations: the $m=1$ case}

In this section we start harvesting the results we can now immediately obtain given this picture of basic hypergeometric functions as faces of the polytope $P^{(1)}$. This was already done for the top two levels (i.e. the functions corresponding to vertices and edges) by Stokman and the authors in [1], though there we did not yet see the polytope. In the next section we give a worked through example (related to ${ }_{2} \phi_{1}$ ) of the abstract results in this section. As a convenient tool to understand the implications of the results mentioned, we refer to Appendix A which contains a list of all the possible functions $B_{\alpha}^{1}$.

Recall that we have a basic hypergeometric function attached to each face of the polytope $P^{(1)}$, and that the Weyl group $W\left(E_{7}\right)$ acts both on $P^{(1)}$ and on sets of parameters $\tilde{\mathcal{H}}_{1}$. As the elliptic hypergeometric function is invariant under this action we immediately obtain

Theorem 6.1. Let $w \in W\left(E_{7}\right), \alpha \in P_{1}$ and $u \in \tilde{\mathcal{H}}_{1}$ then

$$
B_{\alpha}^{1}(u)=B_{w(\alpha)}^{1}(w(u)) .
$$

Proof. Indeed we have

$$
B_{\alpha}^{1}(u)=\lim _{p \rightarrow 0} E^{1}\left(p^{\alpha} \cdot u\right)=\lim _{p \rightarrow 0} E^{1}\left(w\left(p^{\alpha} \cdot u\right)\right)=\lim _{p \rightarrow 0} E^{1}\left(p^{w(\alpha)} \cdot w(u)\right)=B_{w(\alpha)}^{1}(w(u)) .
$$

This gives us formulas of two different kinds for the functions $B_{\alpha}^{1}$.

First of all we can obtain the symmetries of a function by considering the stabilizer of the corresponding face with respect to $W\left(E_{7}\right)$. This includes for example Heine's transformation of $\mathrm{a}_{2} \phi_{1}$, transformations of non-terminating very-well-poised ${ }_{8} \phi_{7}$ 's, and Baileys' four-term relation for very-well-poised ${ }_{10} \phi_{9}$ 's (as a symmetry of a sum of two ${ }_{10} \phi_{9}$ 's).

The symmetry group of the related function is the stabilizer of a generic point in the face, or equivalently the stabilizer of all the vertices of the face. Indeed if some element of $w$ fixes the face, but non-trivially permutes the vertices of the face, then it can be written as the product of a permutation of the vertices generated by reflections in hyperplanes orthogonal to the face, and a Weyl group element which stabilizes the vertices of the face. However, as the functions $B_{\alpha}^{1}(u)$ only depend on the space orthogonal to the face, the first factor has no effect.

Secondly, elements of the Weyl group which send one face to a different face induce transformations relating the two functions associated to these two faces. Examples of this include Nassrallah and Rahman's integral representation of a very-well-poised ${ }_{8} W_{7}$, the expression of this function as a sum of two balanced ${ }_{4} \phi_{3}$ 's, and a relation relating the sum of two ${ }_{3} \phi_{2}$ 's with argument $z=q$ to a single ${ }_{3} \phi_{2}$ with $z=d e / a b c$ [6, (III.34)]. 
Indeed all simplicial faces of $P^{(1)}$ of the same dimension are related by the $W\left(E_{7}\right)$ symmetry, except for dimension 5 . Indeed for dimension 5 there are two orbits: 5 -simplices which bound a 6-simplex and those which do not. In particular in Fig. 2 below, there exist transformation formulas between all functions on the same horizontal level, except for the second-lowest level, where you must distinguish between those faces which are at the boundary of some higher dimensional simplicial face, and those that are not. As two functions between which there exists a transformation formula have the same symmetry group, we have written down the symmetry groups of all the functions on each level on the left hand side. Note that the symmetry group for 5 -simplices at the boundary of a 6 -simplex is 1 (i.e. the group with only 1 element), while for the other 5 -simplices the symmetry group is $W\left(A_{1}\right) \cong S_{2}$.

We can also consider the limit of the contiguous relations satisfied by $E^{1}$. The $q$-contiguous relations reduce to $q$-contiguous relations. We get a relation for each set of three terms $B_{\alpha}\left(u \cdot q^{\beta_{i}}\right)$, where the $\beta_{i}$ are projections of points in the root lattice of $E_{7}$ to the space orthogonal to the face containing $\alpha$.

More interesting is the limit of a $p$-contiguous relation. In order for us to be able to take a limit we have to find three points on $P_{1}$ whose pairwise differences are roots of $E_{7}$.

Proposition 6.2. Let $\alpha, \beta, \gamma \in P^{(1)}$ be such that $\alpha-\beta, \alpha-\gamma, \beta-\gamma \in R\left(E_{7}\right)$ and form an equilateral triangle $($ i.e. $(\alpha-\beta) \cdot(\alpha-\gamma)=1)$, and let $u \in \tilde{\mathcal{H}}_{1}$. Recall $S=\left\{v \in R\left(E_{8}\right) \mid v \cdot \rho=1\right\}$. Then

$$
\begin{gathered}
\prod_{\substack{\delta \in S \\
\delta \cdot(\alpha, \beta, \gamma)=(1,0,0)}}\left(u^{\delta} ; q\right) u^{\gamma} \theta\left(u^{\beta-\gamma} ; q\right) B_{\alpha}^{1}(u)+\prod_{\substack{\delta \in S \\
\delta \cdot(\alpha, \beta, \gamma)=(0,1,0)}}\left(u^{\delta} ; q\right) u^{\alpha} \theta\left(u^{\gamma-\alpha} ; q\right) B_{\beta}^{1}(u) \\
+\prod_{\substack{\delta \in S \\
\delta \cdot(\alpha, \beta, \gamma)=(0,0,1)}}\left(u^{\delta} ; q\right) u^{\beta} \theta\left(u^{\alpha-\beta} ; q\right) B_{\gamma}^{1}(u)=0 .
\end{gathered}
$$

Note that (6.1) is written in its most symmetric form. In order to avoid non-integer powers of the constants one should first multiply the entire equation by $u^{-\alpha}$ and use $u^{\rho}=q$.

Proof. Choose $\zeta$ such that $\tilde{\alpha}=\alpha+\zeta, \tilde{\beta}=\beta+\zeta$ and $\tilde{\gamma}=\gamma+\zeta$ are all roots of $E_{7}$. This is possible by choosing $\tilde{\alpha}$ such that $\tilde{\alpha} \cdot(\alpha-\beta)=1$ and $\tilde{\alpha} \cdot(\alpha-\gamma)=1$, and we can always find roots satisfying these two conditions. Now observe that $\tilde{\alpha} \cdot \tilde{\beta} \leq 1$ as inner product of two different roots of $E_{7}$, and that

$$
\tilde{\alpha} \cdot \tilde{\beta}=(\alpha+\zeta) \cdot(\beta+\zeta)=(\alpha+\zeta) \cdot(\alpha+\zeta)+(\alpha+\zeta) \cdot(\beta-\alpha) \geq 2-1=1,
$$

as $\alpha+\zeta \neq-(\beta-\alpha)$ (equality here would imply $\beta+\zeta=0$ ). Thus $\tilde{\alpha} \cdot \tilde{\beta}=1$, and hence $\tilde{\alpha}, \tilde{\beta}$ and $\tilde{\gamma}$ satisfy the conditions of Theorem 3.5. Setting $t=u \cdot p^{\zeta}$ in (3.3) we obtain

$$
\begin{aligned}
& \prod_{\substack{\delta \in S \\
\delta \cdot(\alpha-\beta)=\delta \cdot(\alpha-\gamma)=1}}\left(u^{\delta} p^{\delta \cdot \beta} ; q\right) u^{\gamma} p^{\gamma \cdot \zeta} \theta\left(u^{\beta-\gamma} p^{\zeta \cdot(\beta-\gamma)} ; q\right) E^{1}\left(u \cdot p^{\alpha}\right) \\
& +\prod_{\substack{\delta \in S \\
\delta \cdot(\beta-\alpha)=\delta \cdot(\beta-\gamma)=1}}\left(u^{\delta} p^{\delta \cdot \gamma} ; q\right) u^{\alpha} p^{\alpha \cdot \zeta} \theta\left(u^{\gamma-\alpha} p^{\zeta \cdot(\gamma-\alpha)} ; q\right) E^{1}\left(u \cdot p^{\beta}\right) \\
& +\prod_{\substack{\delta \in S \\
\delta \cdot(\gamma-\alpha)=\delta \cdot(\gamma-\beta)=1}}\left(u^{\delta} p^{\delta \cdot \alpha} ; q\right) u^{\beta} p^{\beta \cdot \zeta} \theta\left(u^{\alpha-\beta} p^{\zeta \cdot(\alpha-\beta)} ; q\right) E^{1}\left(u \cdot p^{\gamma}\right)=0 .
\end{aligned}
$$

for $u \in \tilde{\mathcal{H}}_{1}$. Now we prove a lemma

Lemma 6.3. Let $\alpha, \beta$ and $\gamma$ be as in the Proposition and let $\delta \in S$ satisfy $\delta \cdot(\beta-\gamma)=0$ then $\delta \cdot \beta \geq 0$. Moreover $\zeta \cdot(\beta-\gamma)=0$ for $\zeta$ as in this proof. 
Proof. Recall the bounding inequalities for $P^{(1)}$ given in Proposition 5.8. Note that $\mu=$ $\rho-\gamma+\beta-\delta \in \Lambda\left(E_{8}\right)$ satisfies $\mu \cdot \rho=1$ and $\mu \cdot \mu=4$, thus we get $\beta \cdot(\rho-\gamma+\beta-\delta) \leq 2$ and similarly $\gamma \cdot(\rho+\gamma-\beta-\delta) \leq 2$. Adding these two inequalities and simplifying gives $\delta \cdot(\beta+\gamma) \geq 0$, and as $(\beta-\gamma) \cdot \delta=0$, this implies $\delta \cdot \beta \geq 0$.

Now observe that

$$
\zeta \cdot(\beta-\gamma)=(\tilde{\alpha}-\alpha) \cdot(\beta-\gamma)=\tilde{\alpha} \cdot(\tilde{\beta}-\tilde{\gamma})-\alpha \cdot(\beta-\gamma)=-\alpha \cdot(\beta-\gamma),
$$

where in the last equality we used that $\tilde{\alpha} \cdot \tilde{\beta}=1=\tilde{\alpha} \cdot \tilde{\gamma}$. Thus we need to show that $\alpha \cdot(\beta-\gamma)=0$. By the bounding inequalities we have $\alpha \cdot(\alpha-\beta) \leq 1$, but also

$$
\alpha \cdot(\alpha-\beta)=(\alpha-\beta) \cdot(\alpha-\beta)-\beta \cdot(\beta-\alpha)=2-\beta \cdot(\beta-\alpha) \geq 1
$$

Thus we find $\alpha \cdot(\alpha-\beta)=1$. By symmetry we also have $\alpha \cdot(\alpha-\gamma)=1$. Thus it follows that $\alpha \cdot \beta=\alpha \cdot \gamma$, or $\alpha \cdot(\beta-\gamma)=0$.

The lemma shows that $p^{\gamma \cdot \zeta}=p^{\alpha \cdot \zeta}=p^{\beta \cdot \zeta}$, so we can divide by this term. Using this lemma we see that we can subsequently take the limit $p \rightarrow 0$ in (6.2) directly as the arguments of the $\theta$ functions do not depend on $p$, while the arguments of the $q$-shifted factorials are either independent of $p$ or vanish as $p \rightarrow 0$.

The relations obtained in this way are three-term relations. By the geometry of the polytope the $\alpha, \beta$ and $\gamma$ in the above proposition must be such that the faces they are contained in, are in the same $W\left(E_{7}\right)$ orbit. In particular we can rewrite our three term relation as a relation between three instances of the same function. Thus we get as examples three-term relations for ${ }_{3} \phi_{2}$ 's [6, (III.33)]. Moreover we obtain the six-term relations of ${ }_{10} \phi_{9}$ 's as studied in [7] and [9].

One reason why the $p$-contiguous relations morally should exist on the elliptic level is that the three functions related by $p$-shifts in roots of $E_{7}$ satisfy the same second order $q$-difference equations (after a suitable gauge transformation). In particular we can take the limit of these $q$ difference equations and see that $B_{\alpha}^{1}, B_{\beta}^{1}$ and $B_{\gamma}^{1}$ also satisfy the same second order $q$-difference equations. In a very degenerate case, there exist faces for which to a vector $\alpha$ in that face there exists exactly one root $r \in R\left(E_{7}\right)$ such $\alpha+r \in P^{(1)}$. In particular, while we cannot find a three term relation in this case, we do obtain the second solution of the corresponding $q$-difference equations. In the general case we can obtain the symmetry group of the $q$-difference equations by looking at the stabilizer of the shifted lattice $\alpha+\Lambda\left(E_{7}\right)$ for a generic point $\alpha$ in the face. This stabilizer, the stabilizer of $\alpha$ under the affine Weyl group, is denoted the affine symmetry group in Fig. 2.

\section{$7 \quad$ An extended example: ${ }_{2} \phi_{1}$}

In this section we consider the simplicial face with vertices $w_{01}, w_{02}, v_{67}$ and $v_{57}$. The centroid of this face is the point $\alpha=(-1 / 4,0,0,1 / 4,1 / 4,1 / 2,1 / 2,3 / 4)$, and we find using Proposition 4.2 that the limit can be expressed as

$$
\begin{aligned}
B_{\alpha}^{1}(u) & =\frac{\left(q, u_{3} u_{0}, u_{4} u_{0} ; q\right)}{\left(q / u_{1} u_{2} ; q\right)} \int \theta\left(u_{0} u_{1} u_{2} / z ; q\right) \frac{\left(q z / u_{7} ; q\right)}{\left(u_{0} / z, u_{3} z, u_{4} z ; q\right)} \frac{d z}{2 \pi i z} \\
& =\left(u_{1} u_{2}, q u_{0} / u_{7} ; q\right)_{2} \phi_{1}\left(\begin{array}{c}
u_{0} u_{3}, u_{0} u_{4} \\
q u_{0} / u_{7}
\end{array} ; q, u_{1} u_{2}\right)
\end{aligned}
$$

as long as this series converges (this integral expression for a ${ }_{2} \phi_{1}$ is not very exciting, as it is related to the series by picking up the residues upon moving the integration contour to zero). 
The stabilizer group of this face under the $W\left(E_{7}\right)$ action equals the stabilizer of $\alpha$ (as it should be a permutation of the four vertices of the face). However those reflections in $W\left(E_{7}\right)$ which non-trivially permute the vertices of the face are in roots which are the difference of two vertices, so they will just induce a shift along a vector in the face; as our functions only depend on the space orthogonal to the face, they act as identity on our function (for example they permute $u_{1} \leftrightarrow u_{2}$ or $\left.u_{5} \leftrightarrow u_{6}\right)$. Thus we are only interested in those elements of $W\left(E_{7}\right)$ which leave the four vertices of this face invariant. In particular, this includes (and by Coxeter theory, is generated by) the reflections in the hyperplanes orthogonal to the roots $\left\{ \pm\left(e_{3}-\right.\right.$ $\left.\left.e_{4}\right), \pm\left(\rho-e_{0}-e_{3}-e_{4}-e_{7}\right), \pm\left(\rho-e_{0}-e_{3}-e_{5}-e_{6}\right), \pm\left(\rho-e_{0}-e_{4}-e_{5}-e_{6}\right)\right\}$. These eight roots form the root system of $A_{2} \times A_{1}$, thus the symmetry group of a ${ }_{2} \phi_{1}$ is $W\left(A_{2} \times A_{1}\right)$, or the permutation group $S_{3} \times S_{2}$. For example the reflection $s_{\rho-e_{0}-e_{3}-e_{4}-e_{7}}$ generates the symmetry $u \mapsto\left(u_{0} / s, u_{1} s, u_{2} s, u_{3} / s, u_{4} / s, u_{5} s, u_{6} s, u_{7} / s\right)$, with $s=\sqrt{u_{0} u_{3} u_{4} u_{7} / q}$, or

$$
\begin{aligned}
& \left(u_{1} u_{2}, q u_{0} / u_{7} ; q\right)_{2} \phi_{1}\left(\begin{array}{c}
u_{0} u_{3}, u_{0} u_{4} \\
q u_{0} / u_{7}
\end{array} ; q, u_{1} u_{2}\right) \\
& \quad=\left(u_{0} u_{1} u_{2} u_{3} u_{4} u_{7} / q, q u_{0} / u_{7} ; q\right)_{2} \phi_{1}\left(\begin{array}{c}
q / u_{4} u_{7}, q / u_{3} u_{7} \\
q u_{0} / u_{7}
\end{array} ; q, u_{0} u_{1} u_{2} u_{3} u_{4} u_{7} / q\right)
\end{aligned}
$$

or simplifying we get

$$
(z, c ; q)_{2} \phi_{1}\left(\begin{array}{c}
a, b \\
c
\end{array} ; q, z\right)=(a b z / c, c ; q)_{2} \phi_{1}\left(\begin{array}{c}
c / b, c / a \\
c
\end{array} ; q, a b z / c\right)
$$

which is one of Heine's transformations [6, (III.3)]. Similarly related to $s_{\rho-e_{0}-e_{4}-e_{5}-e_{6}}$ we obtain

$$
(z, c ; q)_{2} \phi_{1}\left(\begin{array}{c}
a, b \\
c
\end{array} ; q, z\right)=(c / a, a z ; q)_{2} \phi_{1}\left(\begin{array}{c}
a, a b z / c \\
a z
\end{array} ;, c / a\right)
$$

another one of Heine's transformations [6, (III.2)]. Together with the permutation swapping a and $b$ (given by $s_{e_{3}-e_{4}}$ ) these two transformations generate the entire symmetry group.

As for transformations to other functions, there are no less than 6 other faces in the $W\left(E_{7}\right)$ orbit of the face containing $\alpha$ up to $S_{8}$ symmetry. The related transformations are given by (after some simplification)

$$
\begin{aligned}
(z, c ; q) & { }_{2} \phi_{1}\left(\begin{array}{c}
a, b \\
c
\end{array} ;, z\right)=(b z, c ; q)_{2} \phi_{2}\left(\begin{array}{c}
b, c / a \\
b z, c
\end{array} ; q, a z\right) \\
& =\frac{(a, b, a b z / c, q ; q)}{2} \int \frac{\left(y^{ \pm 2}, \sqrt{c z} y^{ \pm 1} ; q\right)}{\left(\sqrt{c / z} y^{ \pm 1}, a \sqrt{z / c} y^{ \pm 1}, b \sqrt{z / c} y^{ \pm 1} ; q\right)} \frac{d y}{2 \pi i y} \\
& =\frac{(z, c / b, c / a ; q)}{(c / a b ; q)} 3 \phi_{2}\left(\begin{array}{c}
a b z / c, a, b \\
q a b / c, 0
\end{array} ; q, q\right)+\frac{(a, b, a b z / c ; q)}{(a b / c ; q)} 3_{3} \phi_{2}\left(\begin{array}{c}
z, c / a, c / b \\
q c / a b, 0
\end{array} ; q, q\right) \\
& =\frac{(z, a b z / c, c ; q)}{(b z / c ; q)}{ }_{3} \phi_{2}\left(\begin{array}{c}
c / b, a, 0 \\
q c / b z, c
\end{array} ; q, q\right)+\frac{(c / b, a, b z ; q)}{(c / b z ; q)}{ }_{3} \phi_{2}\left(\begin{array}{c}
z, a b z / c, 0 \\
q b z / c, b z
\end{array} ; q, q\right) \\
& =\frac{(a z, b z, c ; q)}{(a b z ; q)}{ }_{6} W_{5}^{(2)}\left(\frac{a b z}{q} ; a, b, a b z / c ; q, c z\right) \\
& =(q ; q) \int \theta(z / y ; q) \frac{(c y, a b y ; q)}{(a y, b y, c y / z ; q)} \frac{d y}{2 \pi i y} .
\end{aligned}
$$

Let us now consider the three term relations (as limit of $p$-contiguous relations). The points $\beta$ in the polytope with $\alpha-\beta \in \Lambda\left(E_{7}\right)$ in the root lattice of $E_{7}$ are 


\begin{tabular}{|c|c|c|}
\hline$\beta$ & $B_{\beta}^{1}$ & \\
\hline$\left(-\frac{1}{4}, 0,0, \frac{1}{4}, \frac{1}{4}, \frac{1}{2}, \frac{1}{2}, \frac{3}{4}\right)=\alpha$ & $\left(u_{1} u_{2}, q u_{0} / u_{7} ; q\right)_{2} \phi_{1}$ & $\begin{array}{c}u_{0} u_{3}, u_{0} u_{4} \\
q u_{0} / u_{7}\end{array} ; q, u_{1} u_{2}$ \\
\hline$\left(\frac{3}{4}, 0,0, \frac{1}{4}, \frac{1}{4}, \frac{1}{2}, \frac{1}{2},-\frac{1}{4}\right)$ & $\left(u_{1} u_{2}, q u_{7} / u_{0} ; q\right)_{2} \phi_{1}$ & $\begin{array}{c}u_{7} u_{3}, u_{7} u_{4} \\
q u_{7} / u_{0}\end{array} ; q, u_{1} u_{2}$ \\
\hline$\left(\frac{1}{4}, \frac{1}{2}, \frac{1}{2}, \frac{3}{4},-\frac{1}{4}, 0,0, \frac{1}{4}\right)$ & $\left(u_{5} u_{6}, q u_{4} / u_{3} ; q\right)_{2} \phi_{1}$ & $\begin{array}{c}u_{0} u_{4}, u_{7} u_{4} \\
q u_{4} / u_{3}\end{array} ; q, u_{5} u_{6}$ \\
\hline$\left(\frac{1}{4}, \frac{1}{2}, \frac{1}{2},-\frac{1}{4}, \frac{3}{4}, 0,0, \frac{1}{4}\right)$ & $\left(u_{5} u_{6}, q u_{3} / u_{4} ; q\right)_{2} \phi_{1}$ & $\begin{array}{c}u_{0} u_{3}, u_{7} u_{3} \\
q u_{3} / u_{4}\end{array} ; q, u_{5} u_{6}$ \\
\hline
\end{tabular}

Here we can use the balancing condition $\prod_{r} u_{r}=q^{2}$ to rewrite $u_{5} u_{6}$ in terms of the previous parameters. Any three of these four functions now give a three term relation, for example (after simplification)

$$
\begin{aligned}
(b q / c, q / a, c ; q) \frac{a z}{c} \theta(c / a z ; q)_{2} \phi_{1}\left(\begin{array}{c}
a, b \\
c
\end{array} ;, z\right) \\
\quad+\left(b, c / a, q^{2} / c ; q\right) \frac{q}{c} \theta(a z / q ; q)_{2} \phi_{1}\left(\begin{array}{c}
a q / c, b q / c \\
q^{2} / c
\end{array} ; q, z\right) \\
\quad+(a b z / c, c q / a b z, b q / a ; q) \theta(q / c ; q)_{2} \phi_{1}\left(\begin{array}{c}
b, b q / c \\
b q / a
\end{array} ; q, c q / a b z\right)=0 .
\end{aligned}
$$

The affine symmetry group is now given as the extension of the symmetry group by also allowing elements which permute the four ${ }_{2} \phi_{1}$ 's amongst themselves. Indeed the index $\left[W\left(A_{3} \times\right.\right.$ $\left.\left.A_{1}\right): W\left(A_{2} \times A_{1}\right)\right]=4$. It is also the symmetry group of the $q$-difference equations we discuss next.

The $q$-contiguous equations relate three terms of the form $B_{\alpha}^{1}\left(u \cdot q^{\beta}\right)$ where $\beta$ is in the projection $\Lambda_{\alpha}$ of $\Lambda\left(E_{7}\right)$ on the space orthogonal to the face containing $\alpha$. In particular the lattice $\Lambda_{\alpha}$ is generated by ( $\pi$ denotes the orthogonal projection on $\Lambda_{\alpha}$ )

$$
\begin{aligned}
& \pi(0,0,0,1,0,-1,0,0)=(1 / 4,0,0,3 / 4,-1 / 4,-1 / 2,-1 / 2,1 / 4), \\
& \pi(0,0,0,0,1,-1,0,0)=(1 / 4,0,0,-1 / 4,3 / 4,-1 / 2,-1 / 2,1 / 4), \\
& \pi(0,0,0,0,0,1,0,-1)=(1 / 4,0,0,-1 / 4,-1 / 4,1 / 2,1 / 2,-3 / 4), \\
& \pi(0,0,1,0,0,-1,0,0)=(0,1 / 2,1 / 2,0,0,-1 / 2,-1 / 2,0) .
\end{aligned}
$$

If we simplify ${ }_{2} \phi_{1}$ by setting $a=u_{0} u_{3}, b=u_{0} u_{4}, c=q u_{0} / u_{7}$ and $z=u_{1} u_{2}$, these four vectors correspond to multiplying respectively $a, b, c$, or $z$ by $q$. In particular we have a relation for any three sets of parameters where $a, b, c$ and $z$ 's differ by an integer power of $q$. For example using shifts $\pi\left(\rho-e_{2}-e_{4}-e_{5}-e_{6}\right), \pi\left(e_{1}-e_{5}\right)$ and $\pi\left(e_{1}-e_{2}\right)$ (corresponding to $a \mapsto a q, z \mapsto q z$ and doing nothing), we get

$$
-(1-a)_{2} \phi_{1}\left(\begin{array}{c}
a q, b \\
c
\end{array} ; q, z\right)-a_{2} \phi_{1}\left(\begin{array}{c}
a, b \\
c
\end{array} ; q, q z\right)+{ }_{2} \phi_{1}\left(\begin{array}{c}
a, b \\
c
\end{array} ; q, z\right)=0 .
$$

\section{Evaluations: the $m=0$ case}

In the $m=0$ case the general polytope picture is somewhat unsatisfying as a description of the possible limits of the elliptic hypergeometric beta integral evaluation. Indeed there are two issues. First of all the polytope $P^{(0)}$ as described in Section 5 is not the entire polytope for which proper limits exist, indeed we can give a larger polytope for which this is true. Secondly, if we are interested in knowing what the different evaluations on the basic hypergeometric level are, 
it seems more natural to look at $P_{\mathrm{I}}^{(0)}, P_{\mathrm{II}}^{(0)}$ and $P_{\mathrm{III}}^{(0)}$, and consider the faces of these polytopes, instead of looking at $P^{(0)}$.

Let us first consider this second issue. In Section 5 we have actually shown that to each face of $P_{\mathrm{I}}, P_{\mathrm{II}}$ and $P_{\mathrm{III}}$ there is associated a function, which depends only on the space orthogonal to that face. Moreover the iterated limit property holds in these polytopes. If we therefore want to know what the different limit evaluations are, we only have to write down the faces of these three polytopes and the associated functions with their evaluations. As all faces of these polytopes are simplicial, except for the interior of $P_{\mathrm{II}}$, this is a simple combinatorial argument. All faces are listed in the appendix in Fig. 3. For those faces of $P$ which are split in different faces of $P_{\mathrm{I}}, P_{\mathrm{II}}$ and $P_{\mathrm{III}}$ (i.e. the interior and the facets given by $\alpha_{r}+\alpha_{s}+\alpha_{t}+\alpha_{u}=0$ ) the value of the evaluation might be the same on all these different faces of the smaller polytopes, but as the functions are a priori different, we do obtain a different evaluation formula for each of these faces.

Now we look at the larger polytope.

Definition 8.1. Define the extended polytope $P_{\text {ext }}$ to be the polytope given as the convex hull of the vectors $e_{j}(0 \leq j \leq 5)$ and $f_{j}=\rho^{(0)}-2 e_{j}(0 \leq j \leq 5)$.

The bounding inequalities are

Proposition 8.2. The bounding inequalities of $P_{\mathrm{ext}}$ inside the subspace $2 \rho^{(0)} \cdot \alpha=1$ are given by

$$
\alpha_{r}+\alpha_{s} \leq 1, \quad(0 \leq r<s \leq 5) .
$$

Proof. This follows from a calculation as in Proposition 5.7 (though now we have $\left(\begin{array}{l}7 \\ 2\end{array}\right)$ options as we need to take two vectors from seven).

The following proposition follows immediately from the evaluation formula (3.2).

Proposition 8.3. For $\alpha \in P_{\text {ext }}$ the limit (4.1) exists and $B_{\alpha}^{0}(u)$ is the same for each $\alpha$ in a face of $P_{\text {ext }}$ and depends only on $u$ orthogonal to the face containing $\alpha$. Moreover the iterated limit property holds.

The question this immediately raises is to what extent one can give series or integrals corresponding to points in $P_{\text {ext }} \backslash P^{(0)}$. So far we have not been able to give a good description of these limits, let alone a classification. However we expect this is where we have to look for evaluations of bilateral series. In the next section we consider more generally what we expect.

\section{Going beyond the polytope}

As indicated in the previous section there exist proper limits outside the polytopes as described in Section 5. While we only know of the existence of proper limits as in (4.1) outside of $P^{(m)}$ in the case $m=0$, if we let $p$ tend to zero along a geometric progression and rescale the functions we can obtain limits in many more cases. In general these limits will depend on what geometric progression we use for $p$ (unlike in the $m=0$ case). We do not know for which points in $\mathbb{R}^{2 m+6}$ we can take limits in this way but it does seem to provide a very rich extra set of functions. In particular, this seems to be where bilateral series reside. For instance, Chen and $\mathrm{Fu}[2]$ prove $\mathrm{a}_{2} \psi_{2}$ transformation as a limit of (in our notation) a transformation of $B_{\left(w_{01}^{(1)}+w_{02}^{(1)}+v_{67}^{(1)}\right) / 3}^{(\text {, taken }}$ in a direction pointing outside the Hesse polytope.

As an example we consider $m=1$ and a limit along $\alpha=v_{1}+\epsilon v_{2}$ for small $\epsilon>0$ and $v_{1}$ a vertex of $P^{(1)}$ and $v_{2}$ a root of $E_{7}$ with $v_{1} \cdot v_{2}=0$. Note that all of these limits are related to each other by the Weyl group of $E_{7}$ action, so we expect to obtain transformation formulas relating the functions associated to these vectors. Up to permutations we have the following 6 options: 
1. $(0,0,0,0,0,0,1-\epsilon, 1+\epsilon)$;

2. $(-\epsilon / 2,-\epsilon / 2,-\epsilon / 2, \epsilon / 2, \epsilon / 2, \epsilon / 2,1-\epsilon / 2,1+\epsilon / 2)$;

3. $(-\epsilon, 0,0,0,0, \epsilon, 1,1)$;

4. $(-1 / 2,-1 / 2,1 / 2-\epsilon, 1 / 2,1 / 2,1 / 2,1 / 2,1 / 2+\epsilon)$;

5. $(-1 / 2-\epsilon / 2,-1 / 2+\epsilon / 2,1 / 2-\epsilon / 2,1 / 2-\epsilon / 2,1 / 2-\epsilon / 2,1 / 2-\epsilon / 2,1 / 2-\epsilon / 2)$;

6. $(-1 / 2-\epsilon,-1 / 2+\epsilon, 1 / 2,1 / 2,1 / 2,1 / 2,1 / 2,1 / 2)$.

For some of these we can obtain limits as integrals. In the rest of this section we suppose $\epsilon=1 / N$ for some large integer $N$, and $p=x^{N} q^{k N}$ (where $k$ is allowed to vary).

Proposition 9.1. For $\alpha=(0,0,0,0,0,0,1-\epsilon, 1+\epsilon)$ we have

$$
\lim _{k \rightarrow \infty} E_{1}\left(p^{\alpha} \cdot u\right)\left(x u_{7}\right)^{2 k} q^{2\left(\begin{array}{l}
k \\
2
\end{array}\right)}=\prod_{0 \leq r<s \leq 5}\left(u_{r} u_{s} ; q\right) \frac{(q ; q)}{2} \int \frac{\theta\left(x u_{7} z^{ \pm 1} ; q\right)\left(z^{ \pm 2} ; q\right)}{\prod_{r=0}^{5}\left(u_{r} z^{ \pm 1} ; q\right)} \frac{d z}{2 \pi i z} .
$$

Proof. We calculate

$$
\begin{aligned}
& \lim _{k \rightarrow \infty} E_{1}\left(p^{\alpha} \cdot u\right)\left(x u_{7}\right)^{2 k} q^{2\left(\begin{array}{c}
k \\
2
\end{array}\right)} \\
& =\lim _{k \rightarrow \infty}\left(x u_{7}\right)^{2 k} q^{2\left(\begin{array}{c}
k \\
2
\end{array}\right)} \prod_{0 \leq r<s \leq 7}\left(p^{\alpha_{r}+\alpha_{s}} u_{r} u_{s} ; p, q\right) \frac{(p ; p)(q ; q)}{2} \\
& \times \int \frac{\prod_{r=0}^{5} \Gamma\left(u_{r} z^{ \pm 1}\right) \Gamma\left(p^{1-\epsilon} u_{6} z^{ \pm 1}, p^{1+\epsilon} u_{7} z^{ \pm 1}\right)}{\Gamma\left(z^{ \pm 2}\right)} \frac{d z}{2 \pi i z} \\
& =\prod_{0 \leq r<s \leq 5}\left(u_{r} u_{s} ; q\right) \frac{(q ; q)}{2} \lim _{k \rightarrow \infty}\left(x u_{7}\right)^{2 k} q^{2\left(\begin{array}{c}
k \\
2
\end{array}\right)} \\
& \times \int \frac{\theta\left(p^{\epsilon} u_{7} z^{ \pm 1} ; q\right) \prod_{r=0}^{5} \Gamma\left(u_{r} z^{ \pm 1}\right) \Gamma\left(p^{1-\epsilon} u_{6} z^{ \pm 1}, p^{\epsilon} u_{7} z^{ \pm 1}\right)}{\Gamma\left(z^{ \pm 2}\right)} \frac{d z}{2 \pi i z} \\
& =\lim _{k \rightarrow \infty} \prod_{0 \leq r<s \leq 5}\left(u_{r} u_{s} ; q\right) \frac{(q ; q)}{2} \\
& \times \int \frac{\theta\left(x u_{7} z^{ \pm 1} ; q\right) \prod_{r=0}^{5} \Gamma\left(u_{r} z^{ \pm 1}\right) \Gamma\left(p^{1-\epsilon} u_{6} z^{ \pm 1}, p^{\epsilon} u_{7} z^{ \pm 1}\right)}{\Gamma\left(z^{ \pm 2}\right)} \frac{d z}{2 \pi i z} \\
& =\prod_{0 \leq r<s \leq 5}\left(u_{r} u_{s} ; q\right) \frac{(q ; q)}{2} \int \frac{\theta\left(x u_{7} z^{ \pm 1} ; q\right)\left(z^{ \pm 2} ; q\right)}{\prod_{r=0}^{5}\left(u_{r} z^{ \pm 1} ; q\right)} \frac{d z}{2 \pi i z} \text {. }
\end{aligned}
$$

Here we used that all the poles are on the right side of the contour as $p \rightarrow 0$, so we can interchange limit and integral as before. Moreover we used that

$$
\theta\left(p^{\epsilon} y ; q\right)=\theta\left(q^{k} x y ; q\right)=\theta(x y ; q)\left(-\frac{1}{x y}\right)^{k} q^{-\left(\begin{array}{c}
k \\
2
\end{array}\right)}
$$

for $y=u_{7} z$ and $y=u_{7} / z$.

The next two limits are analogous. 
Proposition 9.2. For $\alpha=(-1 / 2,-1 / 2,1 / 2-\epsilon, 1 / 2,1 / 2,1 / 2,1 / 2,1 / 2+\epsilon)$ we have

$$
\begin{aligned}
& \lim _{k \rightarrow \infty} E_{1}\left(p^{\alpha} \cdot u\right)\left(\frac{q u_{7} x^{2}}{u_{0} u_{1} u_{2}}\right)^{k} q^{2}\left(\begin{array}{c}
k \\
2
\end{array}\right)=\left(u_{0} u_{1}, q ; q\right) \prod_{r=0}^{1} \prod_{s=3}^{6}\left(u_{r} u_{s} ; q\right) \\
& \times \int \frac{\left(1-z^{2}\right) \theta\left(u_{0} u_{1} u_{2} / z x, x u_{7} / z ; q\right)\left(q z / u_{3}, q z / u_{4}, q z / u_{5}, q z / u_{6} ; q\right)}{\left(u_{0} z^{ \pm 1}, u_{1} z^{ \pm 1}, u_{3} z, u_{4} z, u_{5} z, u_{6} z ; q\right)} \frac{d z}{2 \pi i z}
\end{aligned}
$$

and for $\alpha=(-\epsilon / 2,-\epsilon / 2,-\epsilon / 2, \epsilon / 2, \epsilon / 2, \epsilon / 2,1-\epsilon / 2,1+\epsilon / 2)$ we have

$$
\begin{aligned}
& \lim _{k \rightarrow \infty} E_{1}\left(p^{\alpha}\right.\cdot u)\left(\frac{q u_{7} x^{2}}{u_{0} u_{1} u_{2}}\right)^{k} q^{2\left(\begin{array}{c}
k \\
2
\end{array}\right)=(q ; q)} \prod_{r=0}^{2} \prod_{s=3}^{5}\left(u_{r} u_{s} ; q\right) \\
& \times \int \frac{\theta\left(u_{0} u_{1} u_{2} / z x, u_{7} x / z ; q\right)\left(q / u_{7} z, q z / u_{6} ; q\right)}{\left(u_{0} / z, u_{1} / z, u_{2} / z, u_{3} z, u_{4} z, u_{5} z ; q\right)} \frac{d z}{2 \pi i z} .
\end{aligned}
$$

Proof. The first integral is a direct limit in the symmetry broken integral (4.3), while the second limit comes from the symmetric integral with $z \rightarrow p^{\epsilon / 2} z$.

For the other three limits we are unable to describe $B_{\alpha}$ using an integral, but we do have series representations of these limits. As in the case of Proposition 4.3 proofs of these limits involve tedious calculations, so we just give a short sketch. As we have found is quite common for series representations outside the polytope, we obtain bilateral series. For example

Proposition 9.3. For $\alpha=w_{01}+\epsilon\left(e_{1}-e_{0}\right)$ that if $\left|u_{0} u_{1}\right|<1$ we have

$$
\begin{gathered}
\lim _{k \rightarrow \infty} E_{1}\left(p^{\alpha} \cdot u\right) x^{2 k} q^{2\left(\begin{array}{c}
k \\
2
\end{array}\right)}\left(\frac{q}{u_{0}^{2}}\right)^{k}=\frac{\left(u_{0} u_{1} ; q\right)}{(q ; q)} \theta\left(u_{0}^{2} / x^{2} ; q\right) \prod_{r=2}^{7}\left(q x / u_{r} u_{0}, q u_{0} / u_{r} x ; q\right) \\
\times{ }_{8} \psi_{8}\left(\begin{array}{c} 
\pm q u_{0} / x, u_{2} u_{0} / x, \ldots, u_{7} u_{0} / x \\
\pm u_{0} / x, q u_{0} / u_{2} x, \ldots, q u_{0} / u_{7} x
\end{array} ; q, u_{0} u_{1}\right)
\end{gathered}
$$

Proof. To obtain this limit we look at the symmetry broken integral (4.4) with two $s_{i}$ specialized, and change the integration variable $z \rightarrow p^{\epsilon-1 / 2} z$. Subsequently we pick up the poles at $z=u_{0} p^{-2 \epsilon} q^{n}$ (for $n=0$ to $n=2 k$ ) and observe that the remaining integral vanishes in the limit. Moreover the absolute value of the summand in the sum of the residues is maximized near $n=k$ and we can show that we can interchange limit and $\operatorname{sum}$ in $\sum_{n=-k}^{k} \operatorname{Res}\left(z=u_{0} p^{-2 \epsilon} q^{n+k}\right)$, giving a bilateral sum.

Note that for $\left|u_{0} u_{1}\right| \geq 1$ we do not have an explicit expression for the limit. The limit does have an analytic extension to the region $\left|u_{0} u_{1}\right| \geq 1$, but we can only prove this by using the Weyl group symmetry to relate the limit to the previous limits obtained.

In the case $\left|u_{0} u_{1}\right|>1$ we could again use the general method of obtaining a limit: discovering where the integrand or residues are maximized, rescaling properly and interchanging limit and sum/integral. In this case it would lead to

$$
\lim _{k \rightarrow \infty} E_{1}\left(p^{\alpha} \cdot u\right) x^{2 k} q^{2\left(\begin{array}{c}
k \\
2
\end{array}\right)}\left(\frac{q}{u_{0}^{3} u_{1}}\right)^{k}=\frac{\prod_{r=2}^{7} \theta\left(u_{r} x / u_{0} ; q\right)}{\theta\left(x^{2} / u_{0}^{2} ; q\right)}{ }_{1} \phi_{0}\left(\begin{array}{c}
u_{0} u_{1} \\
-
\end{array} ; q, \frac{1}{u_{0} u_{1}}\right)
$$

by picking up the residues at $z=u_{0} p^{-\epsilon} q^{n}$ in the same integral as above but with $z \rightarrow p^{-1 / 2} z$ instead of $z \rightarrow p^{\epsilon-1 / 2} z$. Note that the right hand side vanishes by the evaluation formula for a ${ }_{1} \phi_{0}$. Indeed we can also see that this limit vanishes by applying the Weyl group symmetry before taking the limit and observing a factor $\lim _{k \rightarrow \infty}\left(1 / u_{0} u_{1}\right)^{k}=0$ remains after using one of the integral limits above. In particular this shows one has to be careful taking limits in order to obtain something interesting. 
Proposition 9.4. For $\alpha=(-\epsilon, 0,0,0,0, \epsilon, 1,1)$ and $\left|u_{0} u_{5}\right|<1$ we obtain the limit

$$
\begin{aligned}
\lim _{k \rightarrow \infty} E^{1}\left(p^{\alpha} \cdot u\right)\left(\frac{u_{5} u_{6} u_{7}}{u_{0}}\right)^{k} x^{2 k} q^{2\left(\begin{array}{c}
k \\
2
\end{array}\right)}=\theta\left(u_{0} u_{4} / x, u_{0} u_{1} u_{2} u_{3} / x ; q\right) \\
\times \frac{\left(u_{0} u_{5}, u_{1} u_{4}, u_{2} u_{4}, q u_{3} / u_{1}, q u_{3} / u_{2}, q u_{3} / u_{6}, q / u_{3} u_{6}, q u_{3} / u_{7}, q / u_{3} u_{7} ; q\right)}{\left(q / u_{1} u_{2}, q u_{3}^{2}, u_{4} / u_{3} ; q\right)} \\
\quad \times{ }_{8} W_{7}\left(u_{3}^{2} ; u_{1} u_{3}, u_{2} u_{3}, u_{4} u_{3}, u_{6} u_{3}, u_{7} u_{3} ; u_{0} u_{5}\right)+\left(u_{3} \leftrightarrow u_{4}\right) .
\end{aligned}
$$

Moreover for $\alpha=w_{01}+\epsilon\left(\rho-e_{0}-e_{2}-e_{3}-e_{4}\right)$ (without convergence conditions)

$$
\begin{aligned}
& \lim _{k \rightarrow \infty} E^{1}\left(p^{\alpha} \cdot u\right) x^{2 k} q^{2\left(\begin{array}{c}
k \\
2
\end{array}\right)}\left(\frac{u_{5} u_{6} u_{7}}{u_{0}}\right)^{k} \\
& =\frac{\left(u_{0} u_{1} ; q\right) \prod_{r=2}^{4}\left(q x / u_{0} u_{r}, u_{1} u_{r} ; q\right) \prod_{r=5}^{7}\left(q u_{0} / x u_{r}, u_{0} u_{r} ; q\right)}{\left(q, u_{0}^{2} / x, x u_{1} / u_{0} ; q\right)} \\
& \times{ }_{4} \psi_{4}\left(\begin{array}{c}
u_{0}^{2} / x, u_{0} u_{2} / x, u_{0} u_{3} / x, u_{0} u_{4} / x \\
q u_{0} / x u_{1}, q u_{0} / x u_{5}, q u_{0} / x u_{6}, q u_{0} / x u_{7}
\end{array} ; q, q\right) \\
& +\frac{\prod_{r=2}^{4} \theta\left(u_{0} u_{r} / x ; q\right)}{\theta\left(u_{0} / x u_{1} ; q\right)} \prod_{r=5}^{7}\left(q u_{1} / u_{r}, u_{0} u_{r} ; q\right)_{4} \phi_{3}\left(\begin{array}{c}
u_{0} u_{1}, u_{2} u_{1}, u_{3} u_{1}, u_{4} u_{1} \\
q u_{1} / u_{5}, q u_{1} / u_{6}, q u_{1} / u_{7}
\end{array} ; q, q\right) .
\end{aligned}
$$

Proof. For the first limit, use the symmetry broken integral (4.3) with three specializations, shift $z \rightarrow p^{\epsilon} z$ and pick up the residues at $z=p^{\epsilon} q^{n} / u_{3}$ and $z=p^{\epsilon} q^{n} / u_{4}$. We arbitrarily broke the symmetry between $u_{1}$ and $u_{2}$, with $u_{3}$ and $u_{4}$ here to be able to write this as the sum of only two series.

The second limit is obtained by picking up the residues at $z=p^{-3 \epsilon / 2} u_{0} q^{n}$ (for the ${ }_{4} \psi_{4}$ ) and $z=p^{-\epsilon / 2} u_{1} q^{n}$ (for the ${ }_{4} \phi_{3}$ ) in the symmetry broken integral (4.4) with two specializations, with $z \rightarrow p^{-1 / 2+\epsilon}$.

Now we have obtained these limits we can obtain relations for these functions as before. In particular we obtain the symmetries of these functions (the symmetry group, the stabilizer of $\alpha$ in $W\left(E_{7}\right)$, is isomorphic to $W\left(A_{5}\right)$ ), and we obtain transformation formulas relating all these six functions in terms of each other. This includes the formula [6, (III.38)] expressing an ${ }_{8} \psi_{8}$ in two very-well-poised ${ }_{8} \phi_{7}$ 's.

As mentioned before the big difference between the limits inside the polytope and those outside is that inside we do not need to specialize $p$ to a geometric progression. In particular the functions just obtained do depend non-trivially on the parameter $x ; x$ is not just a cosmetic factor necessary to calculate the limit as $w$ was in Proposition 4.4. The simplest way to see this is to specialize to an evaluation formula. In the elliptic beta integral $E^{1}$ we reduce to $E^{0}$ if the product of two parameters equals $p q$. Thus we must find a pair $r, s$ such that $\alpha_{r}+\alpha_{s}=1$ and set $u_{r} u_{s}=q$. This is not possible for all of the limits, but for example in the case $\alpha=$ $(-\epsilon / 2,-\epsilon / 2,-\epsilon / 2, \epsilon / 2, \epsilon / 2, \epsilon / 2,1-\epsilon / 2,1+\epsilon / 2)$ we can set $u_{5} u_{6}=q$. The limit for the evaluation formula works in precisely the same way, and we obtain

$$
\begin{aligned}
& \left(q / u_{0} u_{7}, q / u_{1} u_{7}, q / u_{2} u_{7} ; q\right) \theta\left(q / x u_{3} u_{7}, q / x u_{4} u_{7} ; q\right) \\
& \quad=(q ; q) \prod_{r=0}^{2} \prod_{s=3}^{5}\left(u_{r} u_{s} ; q\right) \int \frac{\theta\left(u_{0} u_{1} u_{2} / z x, u_{7} x / z ; q\right)\left(q / u_{7} z ; q\right)}{\left(u_{0} / z, u_{1} / z, u_{2} / z, u_{3} z, u_{4} z ; q\right)} \frac{d z}{2 \pi i z} .
\end{aligned}
$$

The left hand side is clearly non-trivially dependent on $x$, while the right hand side is the (rescaled) limit for $\alpha$ specialized in $u_{5} u_{6}=q$. 


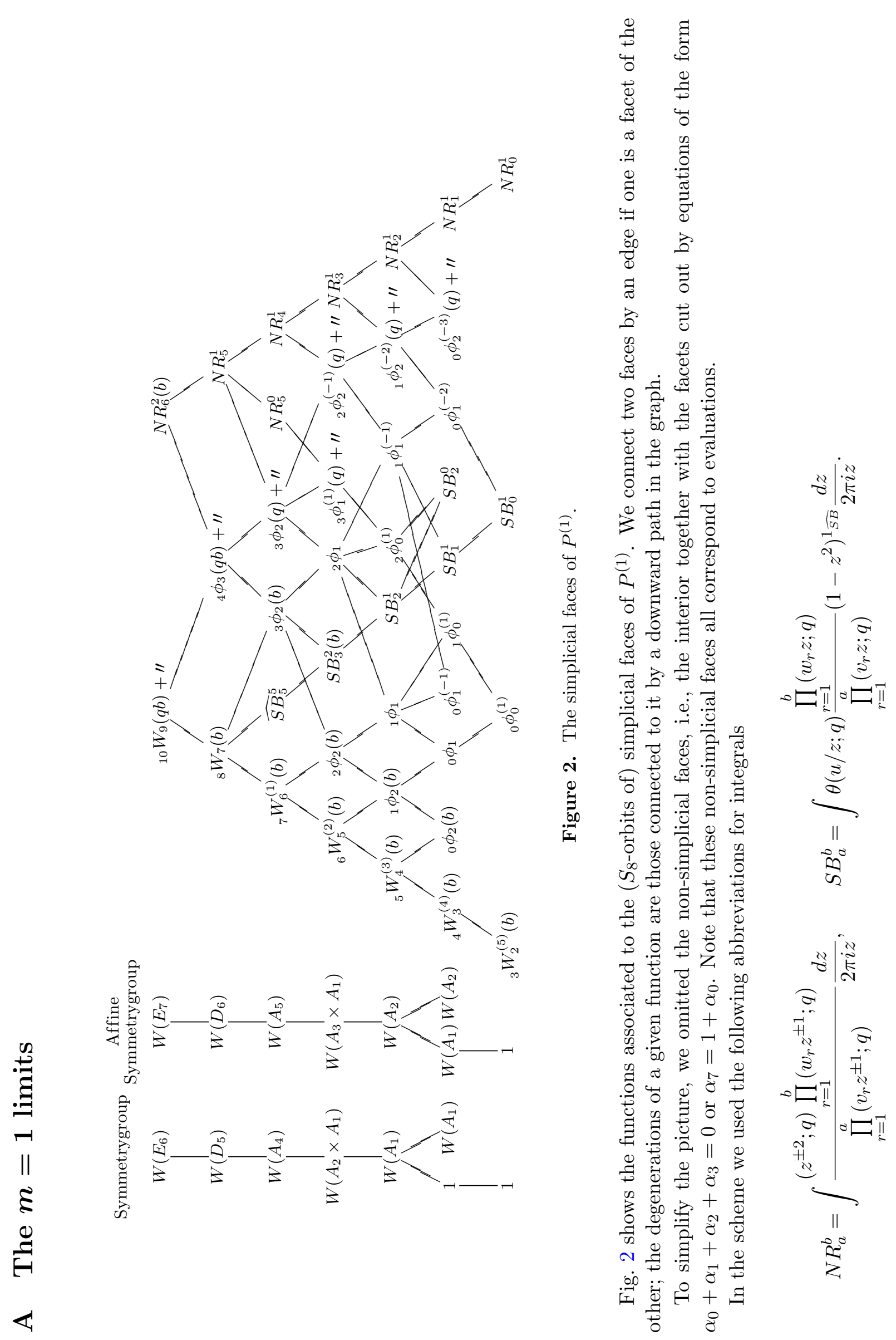


Here $1 \widehat{S B}$ denotes 1 if we consider $\widehat{S B}$ and 0 otherwise. We write ${ }_{r} \phi_{s}+/ /$ or ${ }_{r} W_{r-1}+/$ to denote the sum of two series with related coefficients (as in Proposition 4.2).

After series we write $(q)$ if $z=q$ in the series, $(b)$ if the balancing condition $z \prod a_{r}=\prod b_{r}$ should hold, and $(q b)$ if the series is balanced (i.e. both the above properties hold). Similarly $S_{3}^{2}(b)$ and $N R_{6}^{2}(b)$ indicate that a balancing condition holds amongst their parameters.

We want to stress that all functions are entire in their parameters. In particular for the non-confluent series without the condition $z=q$ (which might fail to converge) we have an integral representation which gives an analytic extension to all values of $z$.

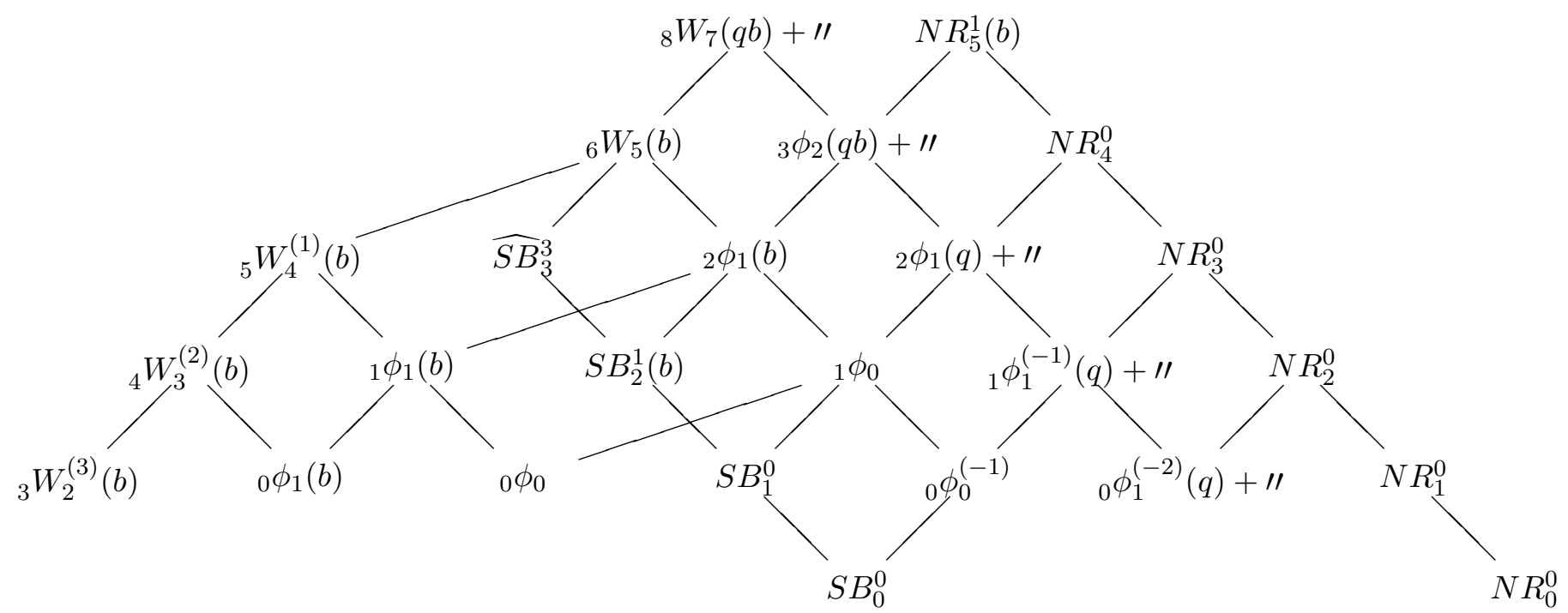

Figure 3. The simplicial faces of $P_{\mathrm{I}}^{(0)}, P_{\mathrm{II}}^{(0)}$ and $P_{\mathrm{III}}^{(0)}$.

In Fig. 3 we have depicted the functions corresponding to the simplicial faces of $P_{\mathrm{I}}^{(0)}, P_{\mathrm{II}}^{(0)}$, $P_{\mathrm{III}}^{(0)}$, i.e. those functions for which we obtain an evaluation formula. The only non-simplicial face of these three polytopes is the interior of $P_{\text {II }}$ (on which the function given by the relevant list of functions, Proposition 4.3, equals 1 identically). The faces of $P_{\mathrm{I}}$ are those which have $N R_{0}^{0}$ as a limit; i.e., the limits of the form $N R_{*}^{*}$. Similarly, the faces of $P_{\mathrm{III}}$ are those which have $S B_{0}^{0}$ as a limit, and any function not of the form $N R_{0}^{0}$ or $S B$ corresponds to a simplicial face of $P_{\mathrm{II}}$. 


\section{Acknowledgements}

The second author was supported in part by NSF grant DMS-0833464.

\section{References}

[1] van de Bult F.J., Rains E.M., Stokman J.V., Properties of generalized univariate hypergeometric functions, Comm. Math. Phys. 275 (2007), 37-95, math.CA/0607250.

[2] Chen W.Y.C., Fu A.M., Semi-finite forms of bilateral basic hypergeometric series, Proc. Amer. Math. Soc. 134 (2006), 1719-1725, math.CA/0501242.

[3] Conway J.H., Sloane N.J.A., The cell structures of certain lattices, in Miscellanea Mathematica, Springer, Berlin, 1991, 71-107.

[4] van Diejen J.F., Spiridonov V.P., An elliptic Macdonald-Morris conjecture and multiple modular hypergeometric sums, Math. Res. Lett. 7 (2000), 729-746.

[5] van Diejen J.F., Spiridonov V.P., Elliptic Selberg integrals, Internat. Math. Res. Notices 2001 (2001), no. 20, $1083-1110$.

[6] Gasper G., Rahman M., Basic hypergeometric series, 2nd ed., Encyclopedia of Mathematics and Its Applications, Vol. 96, Cambridge University Press, Cambridge, 2004.

[7] Gupta D.P., Masson D.R., Contiguous relations, continued fractions and orthogonality, Trans. Amer. Math. Soc. 350 (1998), 769-808, math.CA/9511218.

[8] Lievens S., Van der Jeugd J., Invariance groups of three term transformations for basic hypergeometric series, J. Comput. Appl. Math. 197 (2006), 1-14.

[9] Lievens S., Van der Jeugd J., Symmetry groups of Bailey's transformations for ${ }_{10} \phi_{9}$-series, J. Comput. Appl. Math. 206 (2007), 498-519.

[10] Rains E.M., Transformations of elliptic hypergeometric integrals, Ann. Math., to appear, math.QA/0309252.

[11] Rains E.M., Limits of elliptic hypergeometric integrals, Ramanujan J., to appear, math.CA/0607093.

[12] Rains E.M., Elliptic Littlewood identities, arXiv:0806.0871.

[13] Ruijsenaars S.N.M., First order analytic difference equations and integrable quantum systems, J. Math. Phys. 38 (1997), 1069-1146.

[14] Spiridonov V.P., On the elliptic beta function, Uspekhi Mat. Nauk 56 (2001), no. 1 (337), 181-182 (English transl.: Russian Math. Surveys 56 (2001), no. 1, 185-186).

[15] Spiridonov V.P., Theta hypergeometric integrals, Algebra i Analiz 15 (2003), 161-215 (English transl.: St. Petersburg Math. J. 15 (2004), 929-967), math.CA/0303205.

[16] Spiridonov V.P., Classical elliptic hypergeometric functions and their applications, Rokko Lect. in Math., Vol. 18, Kobe University, 2005, 253-287, math.CA/0511579.

[17] Spiridonov V.P., Short proofs of the elliptic beta integrals, Ramanujan J. 13 (2007), 265-283, math.CA/0408369.

[18] Spiridonov V.P., Essays on the theory of elliptic hypergeometric functions, Uspekhi Mat. Nauk 63 (2008), no. 3, 3-72 (English transl.: Russian Math. Surveys 63 (2008), no. 3, 405-472), arXiv:0805.3135. 